\title{
Equiluminance Cells in Visual Cortical Area V4
}

\author{
Brittany N. Bushnell, ${ }^{1}$ Philip J. Harding, ${ }^{1}$ Yoshito Kosai, ${ }^{1}$ Wyeth Bair, ${ }^{1,2}$ and Anitha Pasupathy ${ }^{1}$ \\ ${ }^{1}$ Department of Biological Structure and Washington National Primate Research Center, University of Washington, Seattle, Washington 98195, and \\ ${ }^{2}$ Department of Physiology, Anatomy, and Genetics, University of Oxford, Oxford OX1 3PT, United Kingdom
}

We report a novel class of V4 neuron in the macaque monkey that responds selectively to equiluminant colored form. These "equiluminance" cells stand apart because they violate the well established trend throughout the visual system that responses are minimal at low luminance contrast and grow and saturate as contrast increases. Equiluminance cells, which compose $\sim 22 \%$ of V4, exhibit the opposite behavior: responses are greatest near zero contrast and decrease as contrast increases. While equiluminance cells respond preferentially to equiluminant colored stimuli, strong hue tuning is not their distinguishing feature-some equiluminance cells do exhibit strong unimodal hue tuning, but many show little or no tuning for hue. We find that equiluminance cells are color and shape selective to a degree comparable with other classes of V4 cells with more conventional contrast response functions. Those more conventional cells respond equally well to achromatic luminance and equiluminant color stimuli, analogous to color luminance cells described in V1. The existence of equiluminance cells, which have not been reported in V1 or V2, suggests that chromatically defined boundaries and shapes are given special status in V4 and raises the possibility that form at equiluminance and form at higher contrasts are processed in separate channels in V4.

\section{Introduction}

Responses of neurons in primate area V4, an intermediate stage along the ventral visual pathway, are sensitive to the color (Zeki, 1973; Schein and Desimone, 1990) and the achromatic luminance of visual stimuli (Schein and Desimone, 1990). But, because studies seldom vary chromaticity and luminance simultaneously, we do not yet know how luminance and chromatic signals are multiplexed in area V4. This is an important question to address because (1) V4 is known to play an important role in form processing (Desimone and Schein, 1987; Kobatake and Tanaka, 1994); (2) in natural vision, form can be defined by chromatic contrast, luminance contrast, or both (Hansen and Gegenfurtner, 2009); and (3) psychophysical studies suggest that both chromatic and luminance contrasts contribute to form perception (Cavanagh, 1991). Deciphering how luminance and chrominance signals are combined across the V4 population will advance our understanding of how cortex produces a unified, invariant perception of form.

Evidence from early stages of visual processing suggests that chrominance-defined and luminance-defined form information is carried by the same neurons and that the influence of lumi-

Received April 14, 2011; revised June 25, 2011; accepted July 8, 2011.

Author contributions: P.J.H., W.B., and A.P. designed research; B.N.B., P.J.H., Y.K., and A.P. performed research; A.P. analyzed data; W.B. and A.P. wrote the paper.

This work was supported by National Eye Institute Grant R01 EY018839, The Whitehall Foundation, University of Washington Vision Core Grant P30 EY01730, and National Center for Research Resources Grant RR00166. W.B. is supported by the Wellcome Trust and St. John's College, Oxford (Oxford, UK). We thank Yasmine El-Shamayleh, Greg Horwitz, and Raghu Pasupathy for helpful discussions and comments. Jalal Baruni and National Primate Research Center Bioengineering provided technical support.

Correspondence should be addressed to Anitha Pasupathy, Department of Biological Structure and Washington National Primate Research Center, University of Washington, 1959 Pacific Street Northeast, HSB G-520, University of Washington Mailbox 357420, Seattle, WA 98195. E-mail: pasupat@u.washington.edu.

DOI:10.1523/JNEUROSCI.1890-11.2011

Copyright $\odot 2011$ the authors $\quad 0270-6474 / 11 / 3112398-15 \$ 15.00 / 0$ nance contrast on chromatic responses is typically facilitatory. Most neurons in V1, V2, and V3 are sensitive to both luminance and color (Gouras and Kruger, 1979; Thorell et al., 1984; Hubel and Livingstone, 1990; Lennie et al., 1990; Johnson et al., 2001; Hansen and Gegenfurtner, 2007), and the contrast response functions in these areas show a characteristic V-shaped pattern (i.e., responses increase, and saturate, with increasing luminance contrasts) (Sclar et al., 1990; Albrecht, 1995; Gegenfurtner et al., 1997; Kiper et al., 1997). A small fraction of V1 neurons respond more strongly to equiluminant stimuli than to luminance stimuli, but because these neurons are typically low pass and unoriented (Lennie et al., 1990; Johnson et al., 2001), they are unlikely to contribute to form encoding. The V1 and V2 neurons equipped to encode object boundaries multiplex luminance and color signals (Lennie et al., 1990; Johnson et al., 2001; Shapley and Hawken, 2002; Hansen and Gegenfurtner, 2007).

To discover how luminance contrast modulates the responses of V4 neurons to colored stimuli, we studied the responses of single neurons in awake monkeys to a set of 25 chromaticities presented at four different luminance contrasts. As in V1, a majority of V4 neurons responded to both chromatic and achromatic stimuli, and luminance contrast had a facilitatory influence on chromatic responses. For a sizable minority, however, equiluminant color stimuli evoked the strongest responses, which decreased when luminance contrasts were added to the chromatic stimuli. These equiluminance cells do not fall into previously established cell classes in V1 or V2, and their shape-selective properties suggest that these neurons can contribute to the encoding of equiluminant colored form.

\section{Materials and Methods}

\section{Surgery and animal behavior}

Two rhesus monkeys (Macaca mulatta; one male and one female) were surgically implanted with custom-built head posts attached to the skull 
with orthopedic screws. After fixation training (see below), a recording chamber was implanted based on structural MRI scans. A craniotomy was performed in a subsequent surgery. For detailed surgical procedures, see Bushnell et al. (2011). All animal procedures conformed to NIH guidelines and were approved by the Institutional Animal Care and Use Committee at the University of Washington.

Animals were seated in front of a computer monitor at a distance of 57 $\mathrm{cm}$ and were trained to fixate a $0.1^{\circ}$ white dot within $0.5-0.75^{\circ}$ of visual angle. Eye position was monitored using a $1 \mathrm{kHz}$ infrared eye-tracking system (Eyelink 1000; SR Research). Stimulus presentation and animal behavior were controlled by Linux-based custom software (PYPE; originally developed in the Gallant Laboratory, University of California, Berkeley, Berkeley, CA). Each trial began with the presentation of a fixation spot at the center of the screen. Once fixation was acquired, four to six stimuli were presented in succession, each for $300 \mathrm{~ms}$ (500 ms for 22 cells), separated by interstimulus intervals of $200 \mathrm{~ms}$. Stimulus onset and offset times were based on photodiode detection of synchronized pulses in the lower left corner of the monitor.

\section{Data collection}

During each recording session, a single dura-puncturing microelectrode (FHC), $250 \mu \mathrm{m}$ in diameter, was lowered into the cortex using an eightchannel acute microdrive system (Gray Matter Research). Signals from the electrode were amplified and filtered and single-neuron activity was isolated using a spike sorting system (Plexon Systems).

We targeted dorsal V4 and our electrode penetrations were largely in the prelunate gyrus and occasionally in the adjoining bank of the lunate sulcus. Previous studies suggest that color selective units are clustered in patches, recently termed "globs" (Conway et al., 2007). While the precise location of globs varies across animals, combining fMRI and physiology, Conway and colleagues suggested that globs were less prevalent in dorsal $\mathrm{V} 4$. We do not know the precise location of globs in our animals, but $15-20 \%$ of our recorded neurons had hue tuning (see Results) that was roughly consistent with that attributed to glob regions.

\section{Visual stimuli}

Visual stimuli were presented on a CRT monitor $(40.6 \times 30.5 \mathrm{~cm} ; 97 \mathrm{~Hz}$ frame rate; $1600 \times 1200$ pixels) calibrated with a spectrophotoradiometer (PR650; PhotoResearch). Stimuli were presented against an achromatic gray background (Fig. $1 \mathrm{~A}$, symbol 1) of mean luminance of 5.4 $\mathrm{cd} / \mathrm{m}^{2}$. For each isolated unit, an initial qualitative preferred stimulus (shape, color, orientation) and a rough receptive field (RF) location were identified using a variety of shapes under the experimenter's control. This was followed by an automated RF mapping procedure that presented the initial preferred stimulus in a densely sampled grid; the refined RF center was based on a two-dimensional Gaussian fit to the data.

To characterize tuning for chromaticity and luminance contrast, we studied the responses to a preferred shape presented in 25 chromaticities each at four different luminances $\left(2.7,5.4,8.1\right.$, and $\left.12.1 \mathrm{~cd} / \mathrm{m}^{2}\right)$. The preferred shape was defined as that which evoked the best response during an initial characterization performed under manual control (varying position, orientation, and size) with the following: drifting and flashing bars, ellipses, and a set of shapes (Fig. $1 B$ ) that were designed to explore a range of convex and concave contour features in the context of a study on partial occlusion (Bushnell et al., 2011). The preferred shape was sized such that all parts of the shape were entirely within the RF of the cell under study. The four luminances tested corresponded to (Weber's) contrasts $\left(C_{\text {lum }}\right)$ of $-50,0,50$, and $125 \%$, respectively. The tested colors spanned the full gamut of the monitor and sampled three triangles at increasing distances from the achromatic point (Fig. $1 A$, symbol 1 ) in the CIE color space. Colors 2-7 sampled the innermost triangle and the lowest color contrast band; colors 8-13 sampled the middle triangle and mid-color contrast band; the remaining colors, which lie on the outermost triangle sampled the highest color contrast band in our study. CIE $x y$ coordinates for the background and the vertices of the outermost triangle (nos. 16, 24, and 20) are as follows: background $=(0.33,0.33)$, red $=(0.5,0.32)$, green $=(0.28,0.45)$, and blue $=(0.17,0.09)$. In this experiment, the equiluminance point ( $0 \%$ contrast) corresponds to photometric equiluminance, which may not match the true equiluminance
A

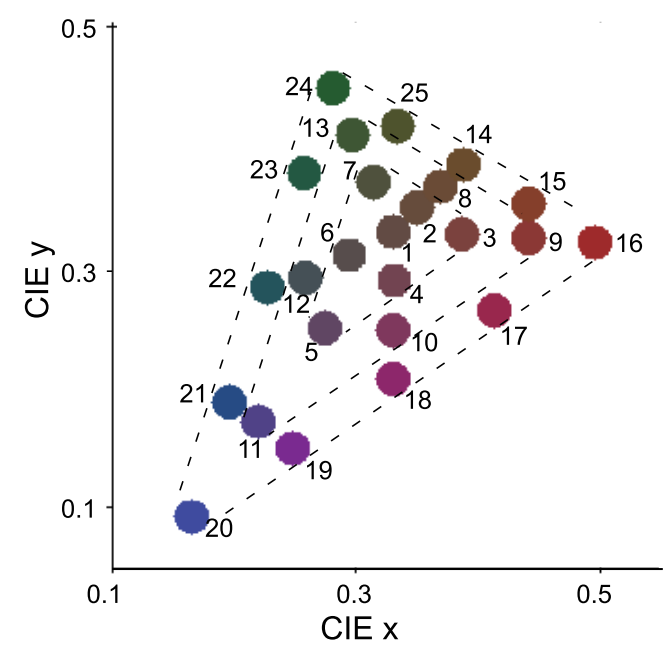

B

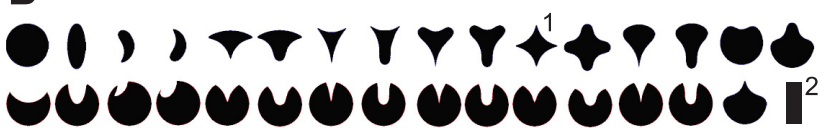

Figure 1. Color and shape stimuli. $\boldsymbol{A}$, The 25 chromaticities used in the study are shown in the CIE chromaticity diagram. The colors occupied three triangles at increasing distances from the achromatic point (no. 1) and represented three levels of color contrast: colors 2-7 composed the low color contrast band; $8-13$, the mid color contrast band; and 14-25, the high color contrast band. Each of the 25 chromaticities was presented at four different luminance contrasts relative to the achromatic background labeled 1. $\boldsymbol{B}$, Shape stimuli used to characterize the shape preferences of neurons. A subset (10-29) of these shapes was presented in eight orientations at $45^{\circ}$ intervals to study shape selectivity. Some shapes that show rotational symmetry were presented at fewer rotations. For example, shapes labeled 1 and 2 were presented at two and four rotations, respectively. Shape labeled 1 was used to characterize color and luminance preferences of example neurons whose data are shown in Figure $2, A, B$, and $D$; shape labeled 2 was used to characterize the example neuron whose data are shown in Figure $2 C$.

point of the animal or the neuron under study. So, for a subset of neurons that responded strongest to $0 \%$ contrast stimuli, we sampled the luminance axis more finely, characterizing responses at $C_{\text {lum }}=0, \pm 2.5, \pm 5$, \pm 25 , and $\pm 50 \%$.

To characterize shape selectivity, for 118 of the 202 neurons, we studied the responses to $10-29$ shapes presented in eight orientations at $45^{\circ}$ intervals in a preferred color based on the color $\times$ luminance test above. Some shapes were tested only at two or four rotations due to rotational symmetry. The tested shapes (Fig. $1 B$ ) were a subset of those previously used to study V4 (Pasupathy and Connor, 2001; Bushnell et al., 2011) and are known to be effective at driving responses of a large fraction of V4 neurons.

\section{Data analysis}

All results presented here are based on mean responses obtained by averaging the firing rate in the interval between stimulus onset and stimulus offset across stimulus repetitions. For each cell, we also computed a latency-adjusted average rate by counting spikes in a window equal to stimulus duration offset by the response latency of the cell at the corresponding luminance contrast. Results based on these latency-adjusted average rates were similar to those reported here. Color and luminance characterizations were based on six pseudorandom repeats, shape results were based on five repeats, and fine-scale sampling along the contrast axis (see Fig. 8) was based on 20 repeats. Responses to the achromatic stimulus at $0 \%$ contrast (blank gray screen) provided a measure of the baseline firing rate. Results presented here are based on mean responses without subtraction of baseline firing, but results were similar with baseline subtraction. 
Contrast response functions. To construct contrast response functions (see Fig. 2, bottom row), for each cell, we first identified colors that evoked responses significantly different from baseline (randomization $t$ test, $p<0.05)$. Then, for each chromaticity that evoked significant responses at one or more luminance contrasts, responses to the different contrasts were normalized to the range $0-1\left[R_{\text {norm }}=R-R_{\min } /\left(R_{\max }-\right.\right.$ $\left.R_{\text {min }}\right)$ ]. The within-chromaticity-normalization ensured that the contrast response functions were not dominated by the colors that evoked the strongest responses. The shape of the contrast response functions was characterized by two sets of parameters: $r_{\text {bright }}$ and $S_{\text {bright }}$, which measured the linear correlation coefficient and slope, respectively, between $C_{\text {lum }} \geq 0$ and the normalized responses, and $r_{\text {dark }}$ and $S_{\text {dark }}$, which measured the same between $C_{\text {lum }} \leq 0$ and the normalized responses. These bright $\left(r_{\text {bright }}\right.$ and $\left.S_{\text {bright }}\right)$ and dark $\left(r_{\text {dark }}\right.$ and $\left.S_{\text {dark }}\right)$ parameters were based on $3 \times n$ and $2 \times n$ data points, respectively, where $n$ represents the number of chromaticities that evoked responses significantly different from baseline for the neuron in question. Mean contrast response functions in Figures 2 and 4 were constructed by averaging the normalized responses across chromaticities.

Separability of the influence of chromaticity and luminance contrast on $V 4$ responses. The validity of pooling contrast response functions across chromaticities, as described above, depends on the separability between the influences of color and luminance contrast on single neuron responses. To directly assess the extent of separability, we used singular value decomposition (SVD), which has been previously used for the same purpose in vision (Mazer et al., 2002) and audition (Depireux et al., 2001; Peña and Konishi, 2001). This method decomposes the color $\times$ luminance contrast response matrix, $R$, of each neuron into the form $U^{T} S V$. $U$ and $V$ are a set of orthogonal vectors and $S$ is a diagonal matrix of singular values; when these are combined, $R$ is fully reconstructed. If the influence of color and luminance are completely separable, only the first diagonal term in $S$ will be nonzero. For every cell, we quantified the singular matrix $S$ and computed a separability index, SI, given by the relative magnitude of the first singular value: $\mathrm{SI}=\alpha(1)^{2} / \Sigma_{i} \alpha(i)^{2}$, where $\alpha(i)$ is the $i$ th diagonal term of $S$. SI values range from 0 for nonseparable matrices to 1 for completely separable matrices. Across our population of V4 neurons, SI was $>0.77$ for all except one cell, and mean \pm SE was $0.93 \pm 0.054$. This indicates that the influence of color and luminance contrast on V4 responses were largely separable. For comparison, in V1 the mean SI for spatial frequency $\times$ orientation was $0.9 \pm 0.09$ (Mazer et al., 2002).

Peristimulus time histograms. To construct population peristimulus time histograms (PSTHs), we first constructed single cell PSTHs at every luminance contrast by averaging and smoothing (Gaussian, $\sigma=5 \mathrm{~ms}$ ) responses across chromaticities. These were normalized by the peak response across all contrasts and then averaged across cells.

Color selectivity. Color selectivity was quantified for each cell in several ways. We used two simple metrics - the number of chromaticities that evoked a response significantly different from baseline (randomization $t$ test, $p<0.05)$ at one or more luminance contrasts $\left(N_{\text {col_sig }}\right)$ and the number of chromaticities that evoked greater than one-half of the maximum response $\left(N_{\text {col_hmax }}\right)$ at the luminance contrast that evoked the best response. We also constructed hue tuning curves at each of the three color contrast bands (Fig. $1 A$ ) by representing the neuronal response as a function of direction in CIE space. We then assessed whether hue tuning was significantly different from uniform tuning using the Rayleigh test of circular uniformity ( $p<0.05$, Bonferroni corrected). Following Conway et al. (2007), strength of unimodal tuning was quantified as the resultant vector length (i.e., the weighted average of the color direction vectors and the corresponding responses). To determine whether color responses of V4 neurons can be modeled as a linear function of cone excitation, for every neuron, we estimated the cone weights, $w_{\mathrm{L}}, w_{\mathrm{M}}, w_{\mathrm{S}}$, that provided the best fit for the observed responses per the following equation:

$$
R=w_{\mathrm{L}} \times C_{\mathrm{L}}+w_{\mathrm{M}} \times C_{\mathrm{M}}+w_{\mathrm{S}} \times C_{\mathrm{S}}+R_{\mathrm{b}},
$$

where $R$ represents the neuronal response; $C_{\mathrm{L}}, C_{\mathrm{M}}$, and $C_{\mathrm{S}}$, the cone contrast signal relative to the background; $w_{\mathrm{L}}, w_{\mathrm{M}}$, and $w_{\mathrm{S}}$, the weights with which the neuron combines these cone signals (Lennie et al., 1990); and $R_{\mathrm{b}}$, the baseline firing rate. To obtain the cone contrasts $\left(C_{\mathrm{L}}, C_{\mathrm{M}}, C_{\mathrm{S}}\right)$ for the various stimuli, the CIE coordinates were first converted to cone excitations based on Smith and Pokorny (1975) fundamentals (Cole and Hine, 1992). Then the cone contrast, $C$, for each of the cone signals was given by the following:

$$
C=\left(E_{\mathrm{stim}}-E_{\mathrm{bg}}\right) / E_{\mathrm{bg}},
$$

where $E_{\text {stim }}$ and $E_{\mathrm{bg}}$ represent the cone excitation for the stimulus and background, respectively. Because some cells responded only to positive or negative contrasts, whereas others responded to both, we implemented half-wave and full-wave rectified versions of the above model (Lennie et al., 1990). For each cell, relative cone weights, $W_{\mathrm{L}}, W_{\mathrm{M}}$, and $W_{\mathrm{S}}$, were obtained by dividing the corresponding absolute cone weights $\left(w_{\mathrm{L}}, w_{\mathrm{M}}, w_{\mathrm{S}}\right.$ from Eq. 1 above) by the sum of the magnitude of the three weights (Lennie et al., 1990).

Shape selectivity. Shape selectivity was characterized with two measures - the fraction of shapes that evoked a significantly different response from baseline $\left(F_{\text {shape_sig }}\right.$; randomization $t$ test, $\left.p<0.05\right)$ and the fraction that evoked greater than one-half of the maximum response $\left(F_{\text {shape_hmax }}\right)$. For shape selectivity, we used the fraction rather than number because different neurons were tested with different numbers of shapes (see Materials and Methods).

\section{Results}

\section{Equiluminance cells in V4}

To examine how luminance contrast modulates neuronal responses to colored shape stimuli in visual area V4, we studied the responses of 202 neurons to a preferred shape presented at 25 chromaticities at each of four different luminance contrasts. Because nearly all neurons in the LGN, V1, V2, V3, V4, and V5/MT show responses that increase, or increase and saturate, as a function of luminance contrast (Sclar et al., 1990; Albrecht, 1995; Gegenfurtner et al., 1997; Kiper et al., 1997; Reynolds et al., 2000; Lee et al., 2007), we expected to find many V4 neurons that increased their responses with luminance contrast, even for colored stimuli. In keeping with this expectation, a majority of V4 cells $(\sim 64 \%)$ in our database showed responses to colored stimuli that increased in magnitude and decreased in latency with increasing luminance contrast. Using example neurons, we first describe three classes of such cells (Fig. $2 A-C$ ), and then introduce a fourth class that exhibited the opposite behavior (Fig. 2D).

Figure $2 \mathrm{~A}$ demonstrates a "bright" cell, which responded best when its preferred pattern (a star rotated by $15^{\circ}$, inset) was brighter than the background. This is evident in the raster plots (top panels), in which raw responses are grouped by luminance contrast. Within each panel, trials are further grouped by color, but this is not apparent because color tuning (analyzed below) was broad for this neuron. The purpose of the raster plots is to illustrate the consistent preference for the positive nonzero contrast across colors. The PSTHs below the rasters summarize the average response time course and show that stimuli brighter than the background $\left(C_{\text {lum }}=50\right.$ and $\left.125 \%\right)$ evoked stronger responses than those equiluminant to $\left(C_{\mathrm{lum}}=0 \%\right)$ or darker $\left(C_{\mathrm{lum}}=-50 \%\right)$ than the background. In addition, the response onset latency decreased progressively with increasing $C_{\text {lum }}$ (see PSTH inset), consistent with previous reports (Gawne et al., 1996; Maunsell et al., 1999). The luminance contrast tuning function (Fig. $2 \mathrm{~A}$, bottom panel) (see Materials and Methods) was characterized by two parameters, $r_{\text {dark }}$ and $r_{\text {bright }}$, which are the correlation coefficients between neuronal responses and $C_{\text {lum }} \leq 0$ or $C_{\text {lum }} \geq 0$, respectively. For this cell, $r_{\text {bright }}$ and $r_{\text {dark }}$ were 0.89 and 0.26 , respectively. Figure $2 B$ illustrates a "dark" cell, which responded preferentially to stimuli (a star at $\left.45^{\circ}\right)$ at negative luminance contrasts $\left(C_{\text {lum }}=\right.$ 

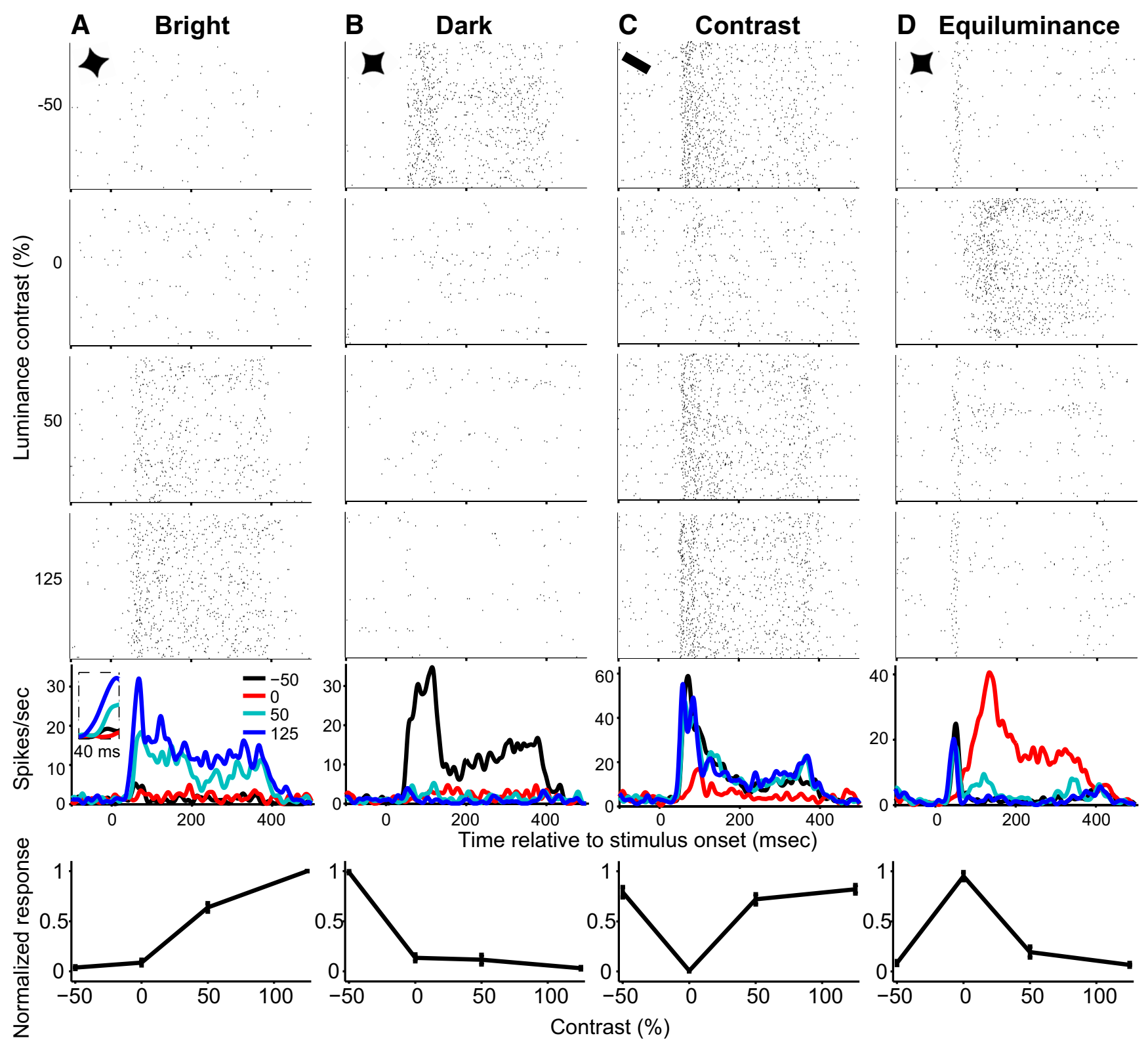

Figure 2. Single neuronal examples of four types of luminance contrast selectivity in V4. $A-D$, Raster plots show single trial responses for six repeats of 25 colors grouped by luminance contrast (rows). Rasters are ordered by color numbering in Figure 1 A. Thus, within each raster plot, rows $1-6$ from the bottom depict responses to color 1 in the CIE diagram, 7-12 represent responses to color 2, etc. The $x$-axis (for rasters and PSTHs) represents time relative to stimulus onset. Stimuli were presented for $300 \mathrm{~ms}$. PSTHs were Gaussian-smoothed with $\sigma=5 \mathrm{~ms}$; line color represents luminance contrast. The inset in $\boldsymbol{A}$ shows enlarged version of the $40 \mathrm{~ms}$ epoch starting $30 \mathrm{~ms}$ after stimulus onset to facilitate response latency comparison across luminance contrasts. Contrast response functions (bottom panel) show mean responses, normalized across colors (see Materials and Methods), versus luminance contrast. Error bars indicate SEM. Example neurons responded strongest to positive luminance contrast (bright cell) $(\boldsymbol{A})$; negative luminance contrast (dark cell) (B); high contrast, either positive or negative (contrast cell) (C); and stimuli that were nominally equiluminant with the background (equiluminance cell) (D).

$-50 \%)$. Stimuli at 0,50 , and $125 \%$ contrasts evoked weak responses. We call this neuron a dark rather than an "off" cell because, while stimuli darker than the background evoked strong responses, offset of bright stimuli did not (see responses to $\left.C_{\text {lum }}=125 \%\right)$. For this neuron, $r_{\text {bright }}$ and $r_{\text {dark }}$ were -0.2 and -0.98 , respectively. Figure $2 C$ shows responses of a "contrast" cell studied with a bar oriented at $60^{\circ}$ relative to the vertical. This neuron responded equally to stimuli at positive and negative contrasts; stimuli at $C_{\text {lum }}=0 \%$ alone evoked weak responses. The contrast tuning function of this neuron was the classical $\mathrm{V}$ shape with $r_{\text {bright }}=0.77$ and $r_{\text {dark }}=-0.91$.

In striking contrast to these examples, Figure $2 D$ shows data from an "equiluminance" cell for which stimuli at $0 \%$ contrast evoked the strongest responses, while stimuli at higher contrasts $(-50,50$, and 125\%) evoked much weaker responses. Unlike results from analogous experiments in V1 (Gouras and Kruger, 1979; Thorell et al., 1984; Hubel and Livingstone, 1990) and V2 (Kiper et al., 1997), the responses of this neuron were suppressed when luminance contrast was added to a patch of color that approximated background luminance. The resulting contrast response function has an inverted $\mathrm{V}$ shape with $r_{\text {bright }}=-0.82$ and $r_{\text {dark }}=0.99$. Even though responses are strongest for $0 \%$ contrast stimuli, the latency at $0 \%$ is longest (red trace), just as in the previous examples. In other words, despite the weaker amplitude, responses to high contrast stimuli emerge earlier than responses to low contrast stimuli (Fig. $2 D$, compare red, blue, and black 
A Bright
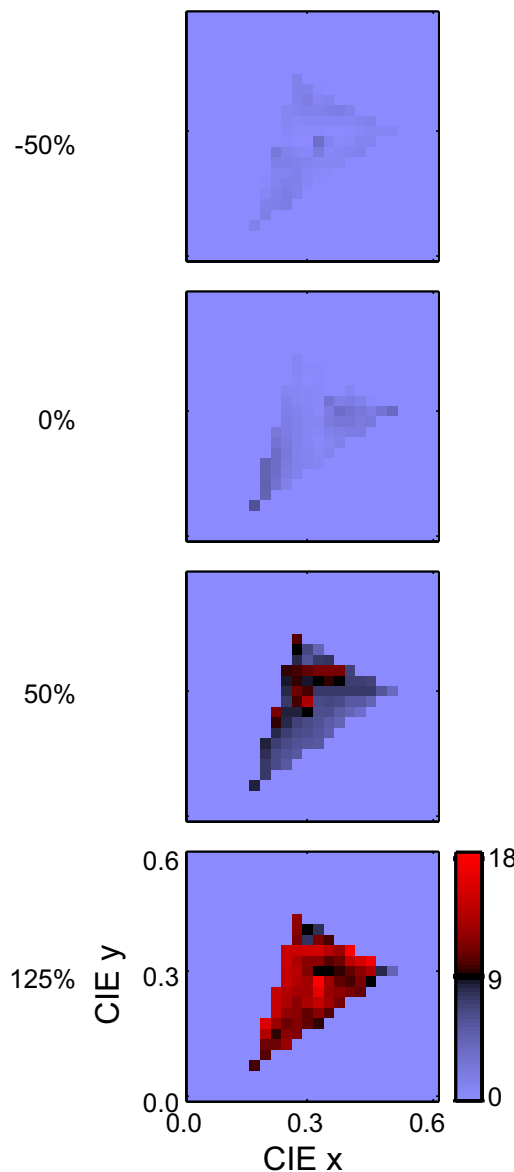

B Dark
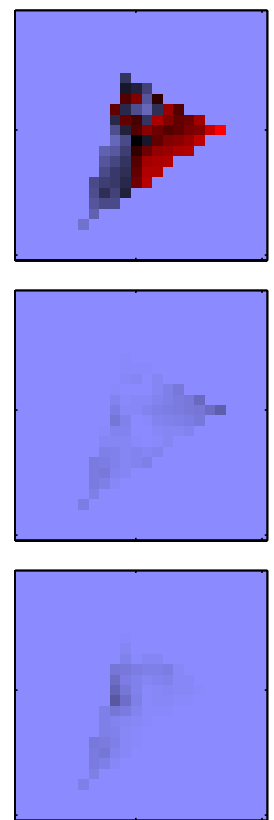

8

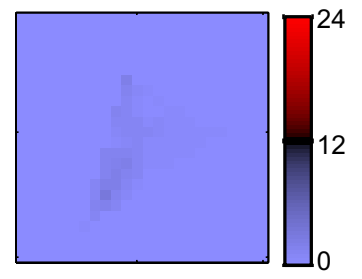

C Contrast
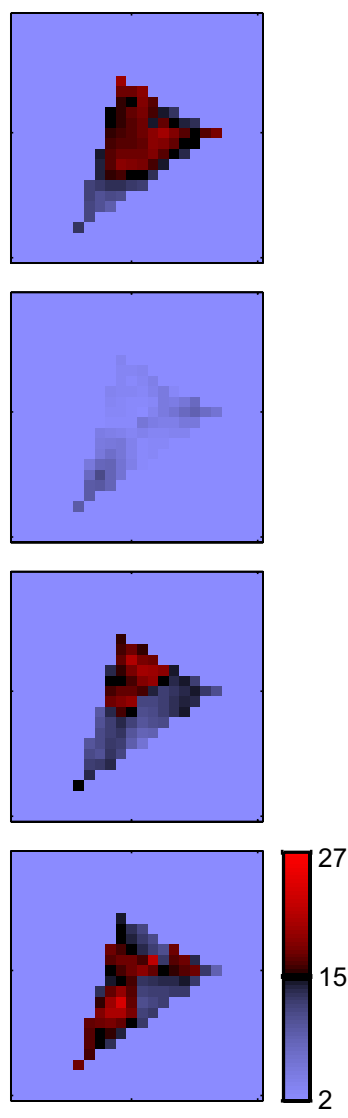

\section{Equiluminance}
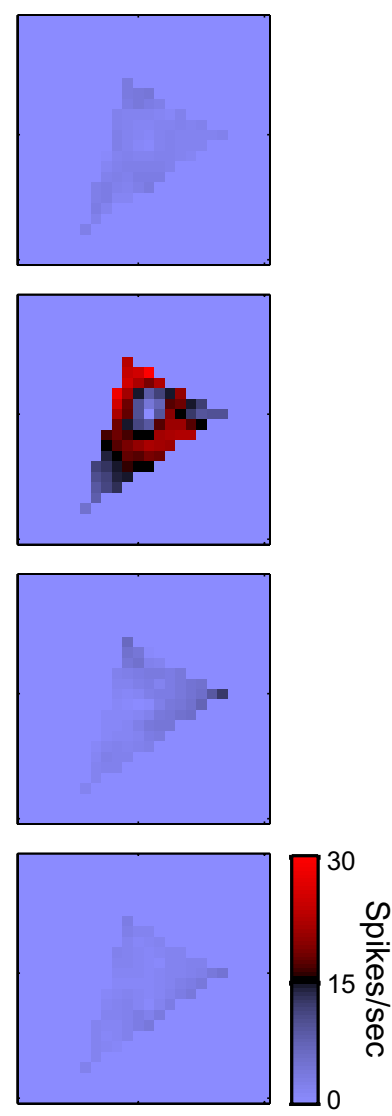

Figure 3. Color preferences and consistency of luminance contrast preference across chromaticities. Mean responses of the example neurons in Figure 2 were linearly interpolated to construct the response surfaces shown here as a function of chromaticity and luminance contrast. Each column depicts an example neuron, and each row represents one of the tested luminance contrasts. For each panel, the CIE $x$ - and CIE $y$-coordinates are plotted along the $x$ - and $y$-axes, and the color scale denotes the strength of the mean response evoked by each stimulus. Mean responses were calculated from stimulus onset to stimulus offset across six presentations. Color scale runs from pale blue (weak responses), through black (intermediate responses), to bright red (strong responses). $\boldsymbol{A}$, Bright cell. Preference for positive luminance contrasts is clearly evident, but color preference is not: most chromaticities at $C_{\text {lum }}=125 \%$ (bottom row) evoked strong responses (red) from this neuron. $\boldsymbol{B}$, Dark cell. This neuron exhibited moderate color tuning with preferred responses clustered close to the red region of the $\mathrm{CIE}$ diagram at $C_{\text {lum }}=-50 \%$. Preference for negative luminance contrasts is consistent across chromaticities. $\boldsymbol{C}$, Contrast cell. There is some clustering of preferred responses (red points) close to the achromatic point; responses at $C_{\text {lum }}=0$ were consistently weak at all chromaticities. $\boldsymbol{D}$, Equiluminance cell. Moderate clustering of preferred responses along the green-magenta axis at $C_{\text {lum }}=0$; responses near the achromatic point, which represents the background color, were weak; this results in an annulus clustering of preferred responses. Responses of all chromaticities, except the background color, were strongest at $C_{\text {lum }}=0$.

PSTHs). This violates the frequently observed relationship between response latency and magnitude, but it supports, in striking fashion, past observations of a dissociation between latency and magnitude in favor of a relationship between latency and luminance contrast (Gawne et al., 1996; Carandini et al., 1997; Maunsell et al., 1999). Put simply, at high luminance contrast, neuronal responses are fast, even if they are weak because of a nonoptimal stimulus form [nonpreferred orientation, as in the study by Gawne et al. (1996)] or a nonoptimal contrast, including high contrasts, for equiluminance cells here.

The contrast response functions in Figure 2 were constructed by pooling across chromaticities (see Materials and Methods), but this would be reasonable only to the extent that the contrast response curves remained qualitatively consistent across chromaticity. To assess consistency for our example cells, and to visualize their color tuning, the responses from the rasters in Figure 2 are replotted in Figure 3 as surfaces in CIE space for each luminance contrast. The bright cell (Fig. $3 A$ ) showed weak color selectivity: at $C_{\text {lum }}=125 \%$, all tested chromaticities evoked significant responses (the number of colors that evoked re- sponses significantly different from baseline, $N_{\text {col_sig }}=25$ ) (see Materials and Methods) and $88 \%$ of colors evoked greater than half-maximum response $\left(N_{\text {col_hmax }}\right.$, number of colors that evoked greater than half-maximum response $=22$ ). Furthermore, a preference for positive luminance contrast is clearly evident and consistent across chromaticities-responses were strongest at $C_{\text {lum }}=125 \%$ for all except one chromaticity [no. 6 (Fig. $1 A$ ) peaks at $C_{\text {lum }}=50 \%$ ] and responses were weaker at $C_{\text {lum }}=-50$ and $0 \%$ for all chromaticities.

The dark cell (Fig. 3B), which responded strongest at $C_{\text {lum }}=$ $-50 \%$, exhibited broad color selectivity $\left(N_{\text {col_sig }}=23 ; N_{\text {col_hmax }}=\right.$ 15) with preferred chromaticities clustered toward red (no. 16) in the CIE space. The effect of luminance contrast was again highly consistent: responses were strongest at $C_{\text {lum }}=-50 \%$ for all except chromaticity no. 12. The contrast cell (Fig. $3 C$ ) responded strongly to all nonzero contrasts, and all colors evoked significant responses at one or more of those contrasts. Color tuning was broad $\left(N_{\text {col } \_ \text {max }}\right.$ was 18 at $C_{\text {lum }}=-50 \%$ and 16 at $C_{\text {lum }}=50$ and $125 \%$ ), and the preferred responses appeared to be clustered in CIE space. All except one of the chromaticities (no. 11) exhibited V-shaped contrast response func- 


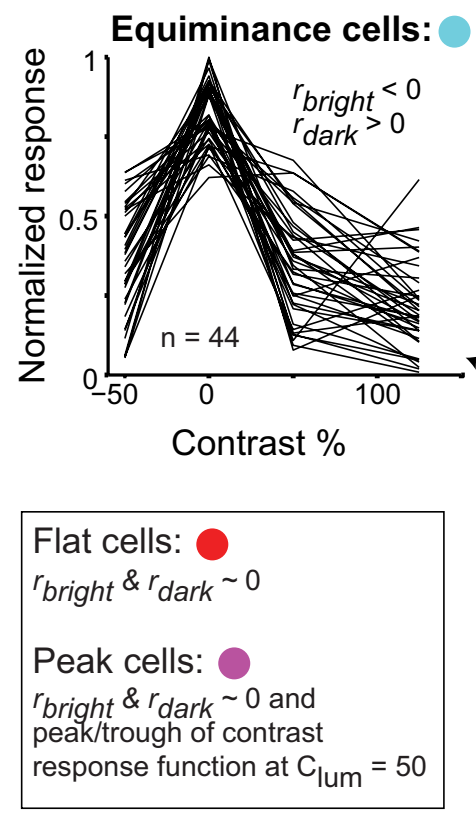

Equiminance cells:

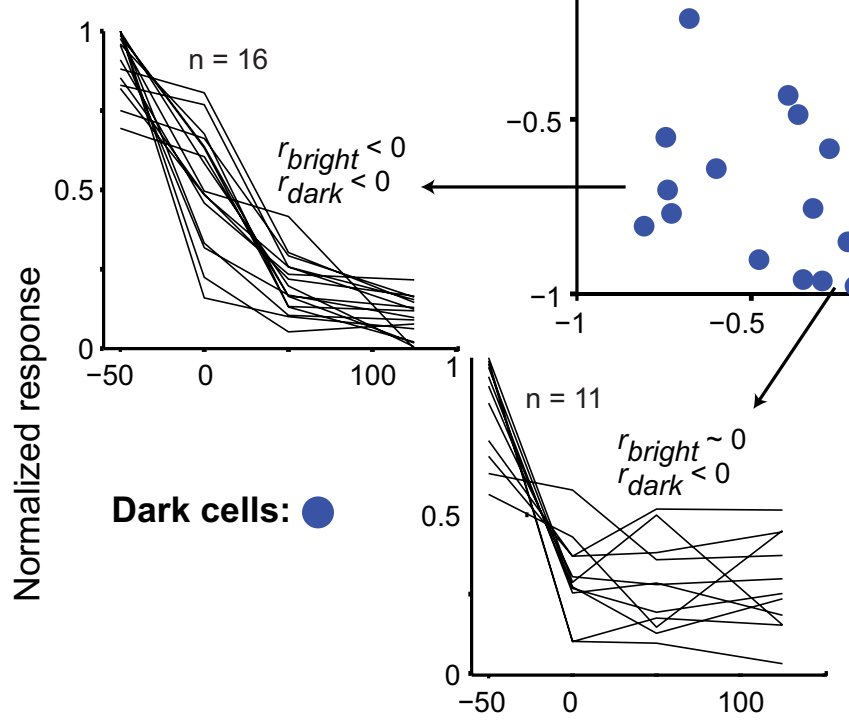

Contrast \%
Bright cells:
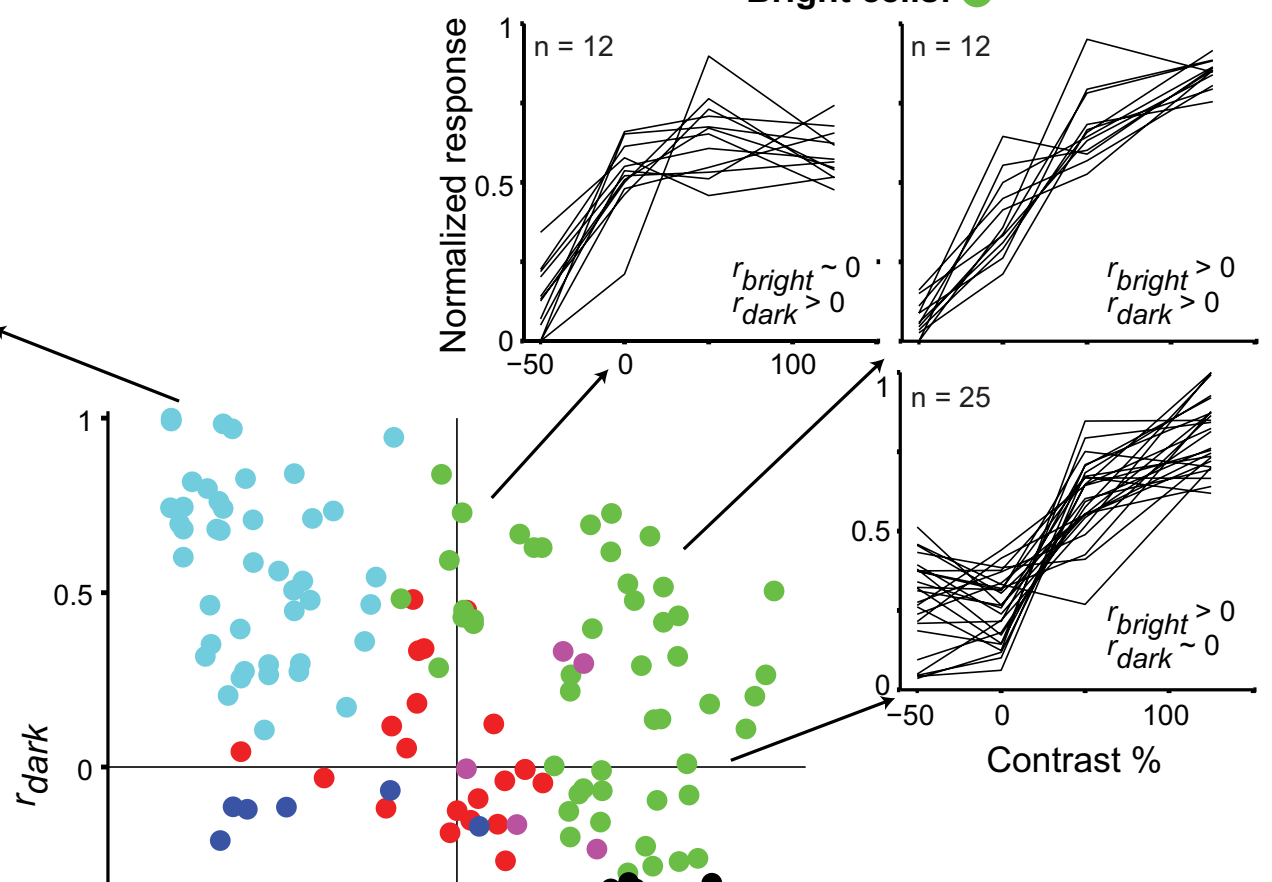

rast \%

Figure 4. Classification of cells based on contrast response functions. Cells were classified into one of six categories (bright, dark, contrast, equiluminance, flat, and peak) based on two correlation values: $r_{\text {bright }}\left(x\right.$-axis of scatterplot), which measures the coefficient of correlation between $C_{\text {lum }}$ and neuronal responses to the different chromaticities for $C_{\text {lum }} \geq 0$, and $r_{\text {dark }}$ ( $y$-axis of scatterplot), which measures the correlation between $C_{\text {lum }}$ and neuronal responses for $C_{\text {lum }} \leq 0$. Only those chromaticities that evoked responses significantly greater than baseline at one or more luminance contrasts were included in the $r_{\text {bright }}$ and $r_{\text {dark }}$ calculations (see Materials and Methods). Mean contrast response functions grouped according to the properties of $r_{\text {bright }}$ and $r_{\text {dark }}$ (listed in the corresponding breakouts; for details, see Results) for bright, dark, contrast, and equiluminance cells are shown in the top right, bottom left, bottom right, and top left respectively. $\sim$ denotes not significantly different from 0 . Number of cells in each breakout panel is indicated by $n$.

tions with minimum response at $C_{\text {lum }}=0 \%$. Finally, the equiluminance cell responded best to chromaticities at $C_{\text {lum }}=0 \%$. Color tuning for this cell was also broad $\left(N_{\text {col_sig }}=22 ; N_{\text {col_hmax }}=13\right)$. Preferred chromaticities ranged from green (no. 24) to magenta (no. 18) with weak responses close to the achromatic point, resulting in an annulus-shaped clustering of preferred responses. All chromaticities except the achromatic point, which is the background color at $C_{\text {lum }}=0 \%$ (and thus no stimulus), exhibited strongest responses at $C_{\text {lum }}=0 \%$.

In summary, these four neurons showed qualitatively consistent trends with luminance contrast across a broad range of chromaticities. To verify this quantitatively, we computed a color luminance separability index using singular value decomposition (see Materials and Methods) and found this index to be $>0.96$ for all four cells, and 0.93 on average across our database. This confirms that the influence of color and luminance contrast were largely separable. Therefore, we will next average across chromaticities to characterize the luminance contrast behavior of our cells. We return later to summarize color tuning in more detail at the end.

\section{Population results}

Figure 4 summarizes the relationship between luminance contrast and neuronal response for 197 of the 202 neurons for which at least two colors evoked responses significantly different from 
baseline (randomization $t$ test, $p<0.05$ ). Based on the shape of the contrast response functions and the extracted parameters $r_{\text {bright }}$ and $r_{\text {dark }}$, cells were classified into one of six categories (indicated by colored symbols in Fig. 4). Bright cells (green dots, 49 of 197; Fig. $2 A$ ) responded weakly to $C_{\text {lum }}<0$ and strongly to $C_{\text {lum }}>0$. Responses at $C_{\text {lum }}=0$ varied across neurons and were either weak and comparable with responses at $C_{\mathrm{lum}}<0$, strong and comparable with responses at $C_{\text {lum }}>0$, or intermediate. This resulted in contrast response functions with three characteristic patterns (Fig. 4 , breakouts in the top right): (1) $r_{\text {bright }}>0, r_{\text {dark }}$ not significantly different from zero (points along the positive $x$-axis, main panel); (2) $r_{\text {dark }}>0, r_{\text {bright }}$ not significantly different from zero (points along the positive $y$-axis); and (3) $r_{\text {bright }}$ and $r_{\text {dark }}>0$ (points in the first quadrant). Dark cells (blue dots, 27 of 197; Fig. $2 B$ ) responded strongest to stimuli that were darker than the background $\left(C_{\text {lum }}<0\right)$ and weakly to $C_{\text {lum }}>0$. At $C_{\text {lum }}=0$, responses were either weak and comparable with responses at $C_{\text {lum }}>0\left(r_{\text {dark }}<0\right.$ and $r_{\text {bright }}$ not significantly different from 0 ; points along the negative $y$-axis), or intermediate between responses to $C_{\text {lum }}>0$ and $C_{\text {lum }}<0\left(r_{\text {bright }}\right.$ and $r_{\text {dark }}<0$, points in the third quadrant). Contrast cells (black dots, 51 of 197), had a V-shaped response profile and responded best to high-contrast stimuli of either polarity. These cells occupied the fourth quadrant $\left(r_{\text {bright }}>0\right.$ and $\left.r_{\text {dark }}<0\right)$. Equiluminance cells (cyan dots, 44 of 197) responded best to stimuli at $C_{\text {lum }}=0$; stimuli brighter or darker than the background evoked weaker responses. Thus, equiluminance cells had inverted V-shaped contrast response functions $\left(r_{\text {bright }}<0\right.$ and $\left.r_{\text {dark }}>0\right)$ and occupied the second quadrant. For the remaining two groups, "flat" (red dots, 21 of 197) and "peak" cells (magenta dots, 5 of 197), $r_{\text {bright }}$ and $r_{\text {dark }}$ were not significantly different from zero. Peak cells, however, showed significant modulation of responses with stimulus contrast and had a peak or trough at $C_{\text {lum }}=50 \%$. These are consistent with peak-shaped luminance response functions in V1 and V2 reported by Peng and Van Essen (2005). The categories depicted here are consistent with those obtained using K-means clustering with number of clusters set to 5 or 6 (data not shown). Results were also similar when we used the contrast response function associated with the chromaticity that evoked strongest responses as the basis for our categorization.

The parameters $r_{\text {bright }}$ and $r_{\text {dark }}$ provide a measure of the consistency of contrast response curves across chromaticities but not of the magnitude of change in response as a function of luminance contrast. Thus, we examined the slopes, $S_{\text {bright }}$ and $S_{\text {dark }}$, between normalized responses and $C_{\mathrm{lum}} \leq 0$ or $C_{\mathrm{lum}} \geq 0$, respectively. Figure $5 \mathrm{~A}$ shows the relationship between the slopes, $S_{\text {bright }}$ and $S_{\text {dark }}$, for the different categories of cells. The spread of slopes is consistent with the spread of correlation coefficients in Figure 4.

While the precise boundaries between categories are not critical, it is important to note several characteristics of the spread of points in Figures 4 and 5A. First, equiluminance cells (cyan) represent a sizable proportion across the population. Results were comparable in both animals (equiluminance cells: monkey $\mathrm{M}$, $21 \%$; monkey C, $24 \%$ ). When the stimuli were presented on a lower background luminance $\left(2.7 \mathrm{~cd} / \mathrm{m}^{2}\right)$, we also found a similar proportion of equiluminance cells ( 16 of $94 \approx 17 \%$ ) across a mostly different set of 94 cells (except 6 cells tested at both background luminances). Second, there is a continuum from equiluminance cells to contrast cells that passes through the origin (flat cells). Third, the spread across all points is not radially symmetric: there is a significant negative correlation $(r=-0.53 ; p<$ $0.001)$ between $S_{\text {bright }}$ and $S_{\text {dark }}$. This suggests that V4 neurons do not evenly cover the space defined by $S_{\text {bright }}$ and $S_{\text {dark }}$; some com-
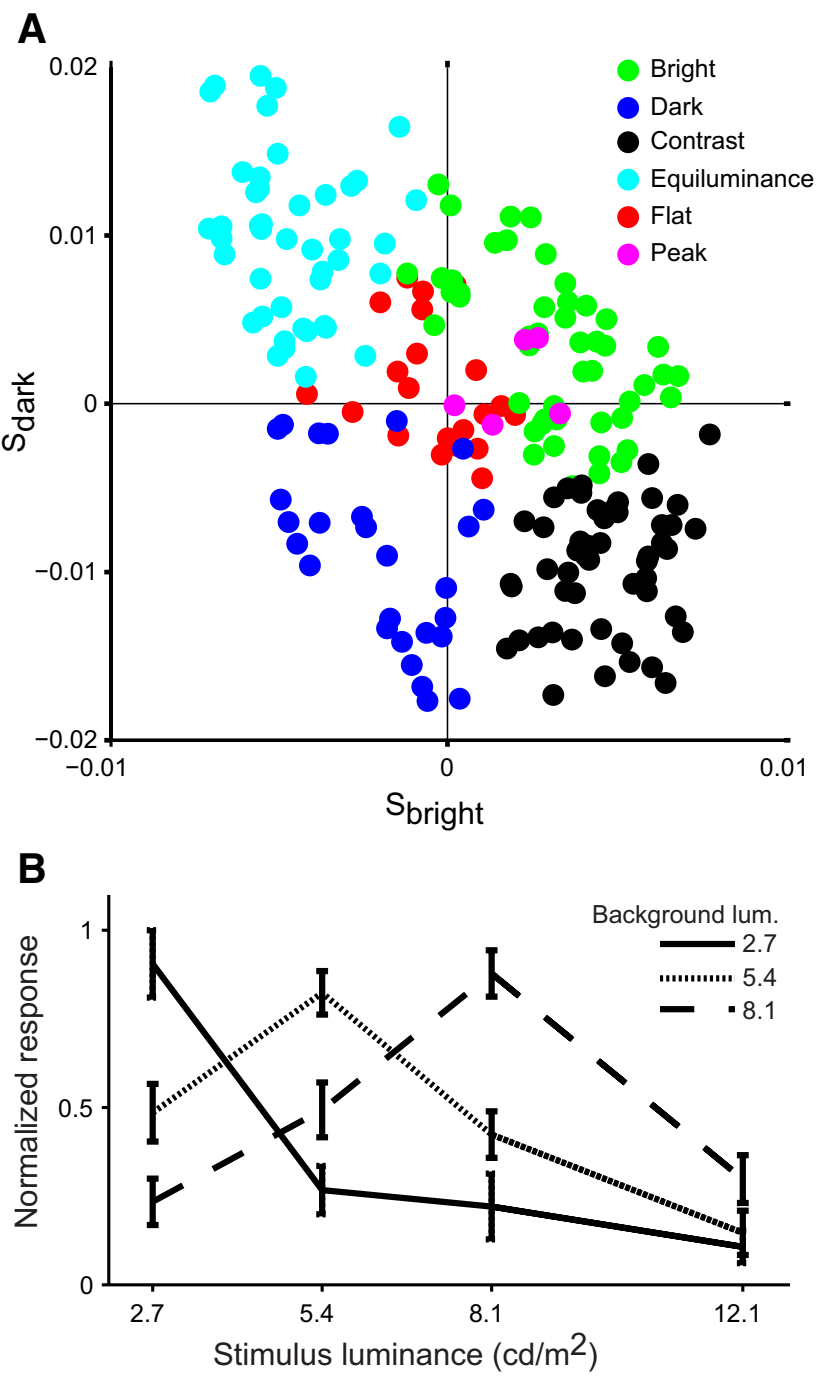

Figure 5. Slopes of the contrast response functions and example cell studied at three background luminances. $A$, Each dot represents a cell. $S_{\text {bright }}(x$-axis of scatterplot) represents the slope given by a linear regression fit between $C_{\text {lum }}$ and neuronal responses to the different chromaticities for $C_{\text {lum }} \geq 0$, and $S_{\text {dark }}$ ( $y$-axis of scatterplot) represents the slope between $C_{\text {lum }}$ and neuronal responses for $C_{\text {lum }} \leq 0$. The dot colors and analysis details are as in Figure 4 . Because the positive contrast range for $C_{\text {Ium }}$ was $0-125 \%$ and the negative contrast range was 0 to $-50 \%$ and responses were normalized to lie between 0 and 1 , the maximum slope limit along the $x$ - and $y$-axes are $0.008(1 / 125)$ and 0.02 (1/50) respectively. Across our population, slopes span this entire range. $\boldsymbol{B}$, Normalized responses ( $y$-axis) plotted as a function of stimulus luminance ( $x$-axis) for an example equiluminance cell studied at three different background luminances. At each background luminance (denoted by line style), individual chromatic contrast response functions were constructed as detailed in Materials and Methods; their mean ( \pm SEM) is plotted here as a function of stimulus luminance rather than contrast. Responses peaked at the stimulus luminance that matched the background luminance, suggesting that luminance contrast, rather than absolute luminance, dictated the response.

binations of $S_{\text {bright }}$ and $S_{\text {dark }}$ are instantiated more often than others. To further test whether equiluminance cells are the result of random variation in the peak position of the contrast response function, we quantified the number of cells with peaks at each of the four tested luminance values. The position of the peak in the mean contrast response function was not evenly distributed between the four tested luminance contrasts $\left(\chi^{2}\right.$ test, $\left.p<0.001\right)$. Peaks at $C_{\text {lum }}=50 \%$, the intermediate positive contrast, were less frequent than peaks at other contrasts, indicating that cells favored peaks at the extreme contrasts, as is common throughout visual cortex, or at $C_{\text {lum }}=0$, which is the novel class of equilu- 


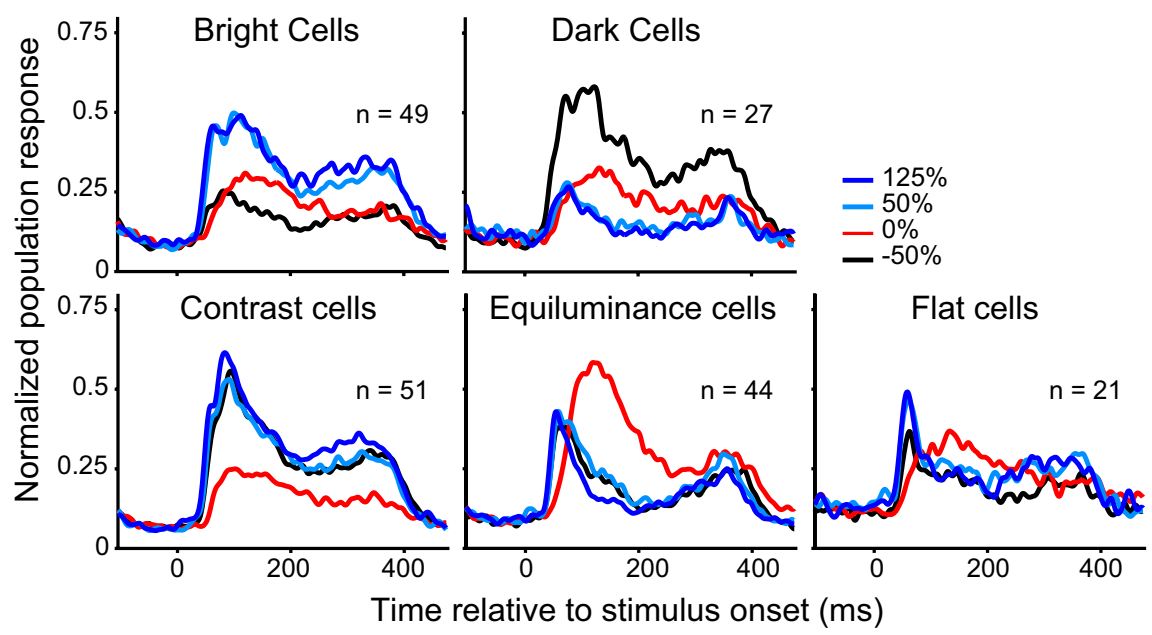

Figure 6. Time course of the population response for each cell class. Population PSTHs show the average normalized response (see Materials and Methods) relative to stimulus onset for bright, dark, contrast, equiluminance, and flat cells. Number of cells contributing to each histogram is indicated by $n$. Because few cells were categorized as peak cells $(n=5)$, the corresponding PSTHs are not shown. Stimulus duration was $300 \mathrm{~ms}$ except for a few cells ( $3-6$ in each category; $500 \mathrm{~ms}$ ). The line color represents stimulus contrast as per the legend. The response to near equiluminant stimuli (red line) was always the latest response, regardless of whether it was the strongest or weakest response on average. As in the examples in Figure 2, bright, contrast, and dark cells responded strongest to high-contrast stimuli, while equiluminance cells responded best to $0 \%$ contrast.

minance cells described here. Thus, equiluminance cells are almost as prevalent as bright or contrast cells in area V4 but more prevalent than cells with peaks at intermediate positive contrasts. This does not argue against equiluminance cells being a part of a continuous distribution of contrast response functions in V4, but it does suggest that they are an important part of that continuum and could represent a specialization in V4 for the representation of equiluminant form (see Discussion).

To control for the unlikely possibility that equiluminance cells prefer the actual background luminance $\left(5.4 \mathrm{~cd} / \mathrm{m}^{2}\right)$ rather than $0 \%$ luminance contrast, we studied three equiluminance cells at two additional background luminances $\left(2.7\right.$ and $\left.8.1 \mathrm{~cd} / \mathrm{m}^{2}\right)$. All details of the experiment were identical except for the background luminance. Results for an example neuron at all three background luminances are shown in Figure $5 B$. The three curves peaked at different luminances depending on the background luminance: the peak position matched the background luminance. This indicates that the neuronal response reflected a preference for the stimulus contrast rather than the luminance itself. All three control neurons showed the same pattern of results. Consistent with this result, if equiluminance cells preferred the actual luminance rather than the $0 \%$ contrast, one would expect the distribution of preferred luminances to be evenly distributed between the four values tested, but this was not the case (see above). These analyses support the hypothesis that luminance contrast, rather than luminance, was the relevant stimulus attribute.

Having found a class of cells that respond best at low luminance contrasts, we examined whether these cells also turn on its head the usual latency relationship with luminance contrast: high-contrast signals typically produce responses that are not only stronger but that also tend to occur sooner. Figure 6 shows the population PSTHs for the different cell categories and the results match the corresponding single cell examples in Figure 2. Bright, dark, and contrast cell populations responded best to high-contrast stimuli, whereas equiluminance cells showed strongest responses at $C_{\text {lum }}=0$. In terms of response latency, however, all cell classes followed a similar trend: latency decreased with increas- ing luminance contrast. In particular, comparing contrast cells to equiluminance cells, the response to $0 \%$ contrast (red lines, bottom row) rises later than the other responses, despite the reversal of the relative amplitudes of the signals. It is interesting to note that the peak times for the high contrast and equiluminance population responses in V4 correspond remarkably well with the optimal stimulus duration for the detection of luminance (55 ms) and chrominance (140 ms) flashes, respectively (Chaparro et al., 1993).

Since responses of equiluminance cells to color stimuli are suppressed in the presence of high luminance contrast, we considered the possibility that high color contrast, just like high luminance contrast, also suppresses the responses of these cells. We constructed population PSTHs with the chromaticities split into three color contrast bands (Fig. 1A) (see Materials and Methods). Figure 7 shows color contrast PSTHs for stimuli at $0 \%$ luminance contrast in shades of red. Across the population of equiluminance cells, peak amplitude is quite similar for all three color contrast bands; specifically, peak amplitude for high color contrast was not weaker than for the lower color contrasts. This suggests that the suppression of equiluminance cell responses is specific to luminance contrast. Interestingly, however, color contrast, like luminance contrast, appears to modulate response latency: responses emerged earlier for stimuli at higher color contrast. The trend in latency as a function of color contrast is not evident for stimuli at a higher luminance contrast (blue lines), presumably because the effect of luminance contrast swamped the more subtle changes due to color contrast. This gives a coherent impression that contrast, whether luminance or color, is a fundamental driver of response strength, as reflected by latency. Nevertheless, equiluminance cells must have specific circuitry to reverse the amplitude relationship, but not the time relationship.

All of the results presented so far are based on photometric equiluminance (i.e., $C_{\text {lum }}=0$ as defined using a photometer and not the behaviorally determined equiluminance points of the animal or that of any given cell). Because the equiluminance point can vary between species, individual subjects, and even between cells, we sampled the contrast axis more finely about photometric equiluminance for 13 equiluminance cells and verified that responses in the -5 to $5 \%$ contrast range were similarly high and showed a gradual decline to baseline levels near $\pm 25 \%$ contrast (Fig. 8). In every case, the chromatic contrast response function (red line) was unimodal with a peak close to $C_{\text {lum }}=0$, and the best achromatic contrast response (black line) was always weaker than the best chromatic response near equiluminance. These results support the conclusion that equiluminance cells respond best to preferred chromaticities near equiluminance and that these responses cannot be attributed to selectivity for low achromatic contrast stimuli.

\section{Comparison with V1}

To examine how our results compare with previous findings in V1, we characterized our cells in terms of their color sensitivity 


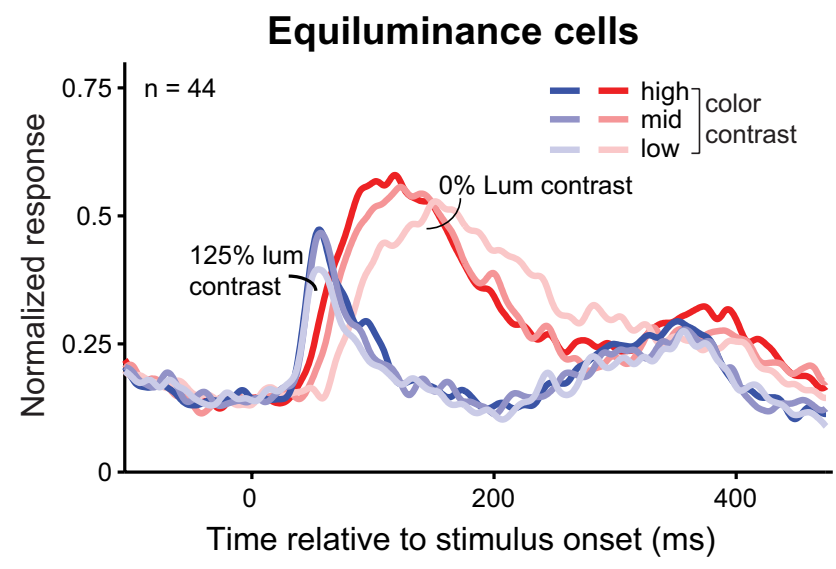

Figure 7. Population PSTHs as a function of color contrast. Stimuli were divided into three groups based on color contrast: chromaticities 2-7 (low color contrast band), 8-13 (mid color contrast band), and 14-25 (high color contrast band). Population PSTHs for equiluminance cells based on these three groups of stimuli are shown for stimuli at $0 \%$ luminance contrast (shades of red) and $125 \%$ luminance contrast (shades of blue). The $x$ - and $y$-axes are as in Figure 6. At $0 \%$ luminance contrast, peak amplitudes are similar for the three color contrasts, suggesting that responses of equiluminance cells to high color contrast are not also suppressed. Responses emerged earlier for higher color contrasts for stimuli at $0 \%$ luminance contrast but not for stimuli at $125 \%$ luminance contrast.

(Johnson et al., 2001) and in terms of a linear cone weight model (Lennie et al., 1990). For each cell, we calculated a color sensitivity index, $\mathrm{SI}=\mathrm{Re} / \mathrm{Ra}$, where $\mathrm{Re}$ is the best response (in excess of baseline) in the equiluminant plane and $\mathrm{Ra}$ is the best response to achromatic stimuli. The distribution of SI is shown in Figure 9A for all cells, arranged by the cell categories defined in Figure 4. The SI values for bright, dark, and contrast cells were centered near 1.0 and most ranged between 0.5 and 2.0, indicating that the best achromatic and equiluminant responses differed by no more than a factor of 2 . This is similar to the range of values exhibited by V1 color luminance cells described by Johnson et al. (2001). Most equiluminance cells, however, had SI $>2.0$ (i.e., the response to the best equiluminant stimulus was at least twice as large as the response to the best achromatic stimulus). A small proportion $(\sim 11 \%)$ of $\mathrm{V} 1$ cells also exhibit indices $>2.0$ (color cells; Johnson et al., 2001). Compared with V1, far fewer neurons had indices $<0.5$, where the best achromatic response was at least twice as large as the best equiluminant response (luminance cells; Johnson et al., 2001). To assess whether this was simply due to the imbalance in our comparison-Re was the maximum of 4 numbers, while Ra was the maximum of 24 numbers - we computed an SI distribution based on the maximum of 3 numbers for each. We redefined $\mathrm{Ra}$ as the maximum response to the three nonzero achromatic contrasts and $\mathrm{Re}$ as the maximum response to the most saturated red, green, and blue in our stimulus set (Fig. $1 \mathrm{~A}$, symbols 16,24 , and 20 ). In this case, $17 \%$ of V4 neurons exhibited values of SI $<0.5$ (up from $5 \%$ in Fig. $9 A$, as expected), but this was still substantially less than the proportion observed in V1 (60\%; Johnson et al., 2001). Further experiments are needed to determine whether this difference between cortical areas reflects a specialization of function, or is simply attributable to the fact that we tested more color directions in the equiluminant plane than Johnson et al. (2001). For the 13 equiluminance cells with finely sampled chromatic and achromatic contrast response functions (Fig. 8), SI values similarly stood apart from the other groups of cells when $\mathrm{Ra}$ and $\mathrm{Re}$ were based on the finely sampled contrast response functions.
A Single cell examples

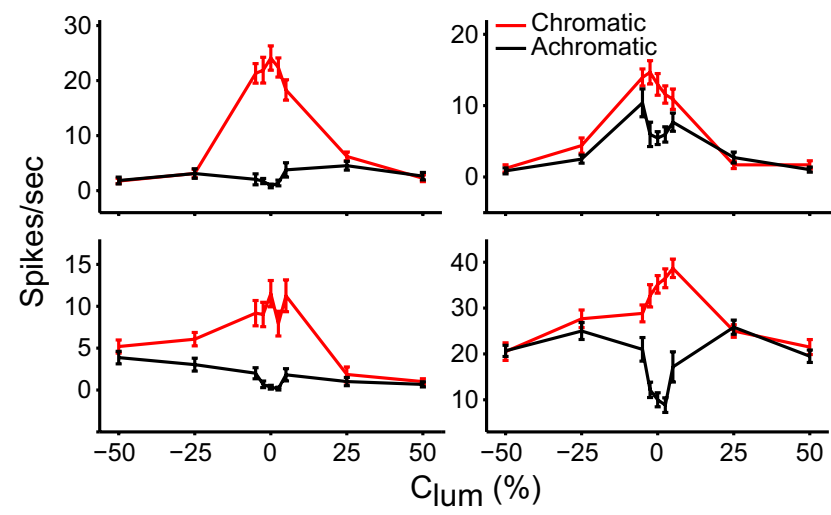

B

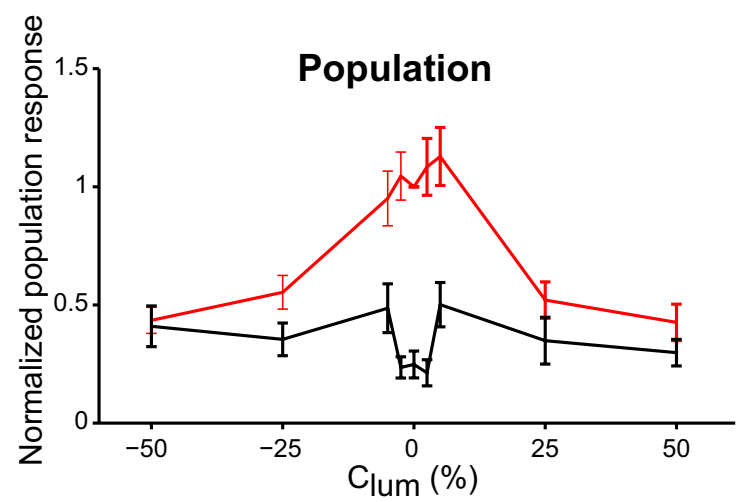

Figure 8. Finer sampling of the contrast response function. $\boldsymbol{A}$, Each panel shows the responses of an equiluminance cell to a preferred chromaticity (red line) and the achromatic stimulus (black line) presented at $C_{\text {lum }}=0, \pm 2.5, \pm 5, \pm 25$, and $\pm 50 \%$ luminance contrasts. Mean responses (across 20 repetitions) are plotted against luminance contrast ( $x$-axis). Error bars represent SEM. In four representative single cells, chromatic responses tended to be high near $0 \%$ contrast and gradually declined for higher contrasts (25-50\%). Responses to achromatic contrasts were typically bimodal with weakest responses at zero and high contrasts. Responses to the best achromatic contrast were weaker than to chromatic stimuli near equiluminance. $\boldsymbol{B}$, Normalized population response across all 13 cells on which the finer sampling was conducted. The responses of each cell were normalized by the response to the chromatic stimulus at $0 \%$ luminance contrast. Thus, at $C_{\text {lum }}=0, y$ for the red line equals 1 by definition. Across the population, chromatic contrast response functions were quite narrow: responses decline to $50 \%$ of the peak at $\pm 25 \%$ contrast. For this range of contrasts, responses to achromatic contrasts were much weaker. Note that the $x$-axes here range from -50 to +50 , unlike in Figures 2 and 4 , in which they range from -50 to +125 .

The observation that equiluminance cells occupy the right tail of the SI distribution in Figure $9 A$ is expected by definition because cells responding best to stimuli in the equiluminant plane and weaker to higher contrasts were classified as equiluminance cells. However, that most contrast cells and a large fraction of bright and dark cells do not occupy the space defined by SI $>2.0$ does not follow from our categorization scheme. A large fraction of these cells are clustered close to $\mathrm{SI}=1.0$ (i.e., $\mathrm{Re} \approx \mathrm{Ra}$ ). Such neurons cannot have balanced cone opponency if their responses arise from linear weighting of cone excitation because balanced opponency in the linear model implies $\mathrm{Re}>\mathrm{Ra}$. For instance, in Equation 1, if $w_{\mathrm{L}}=-w_{\mathrm{M}}$, stimuli in the equiluminant plane with $C_{\mathrm{M}}$ and $C_{\mathrm{L}}$ of opposite sign will evoke greater responses than achromatic stimuli ( $C_{\mathrm{M}}$ and $C_{\mathrm{L}}$ of the same sign).

To determine whether a linear combination of cone excitation signals can explain V4 responses and to assess the sign and magnitude of the corresponding cone weights, for each neuron, we 
A

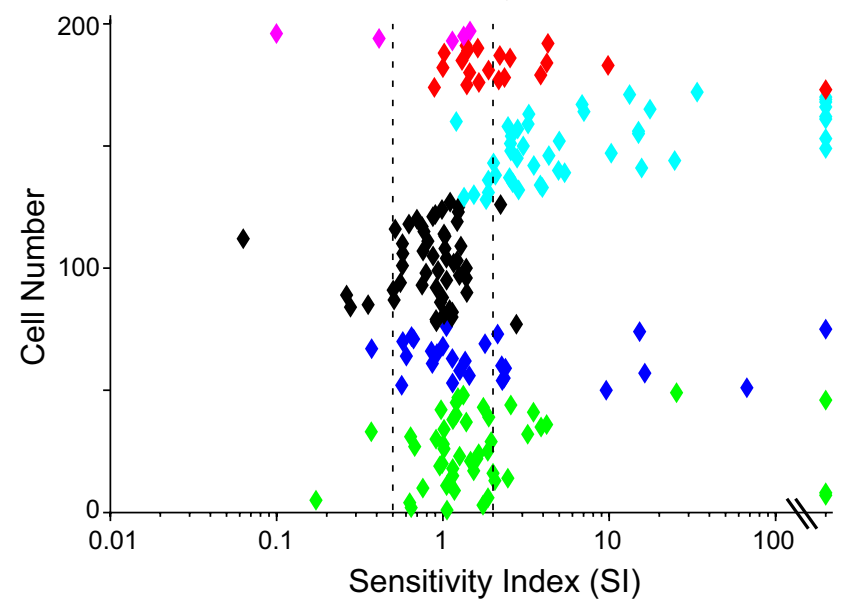

B

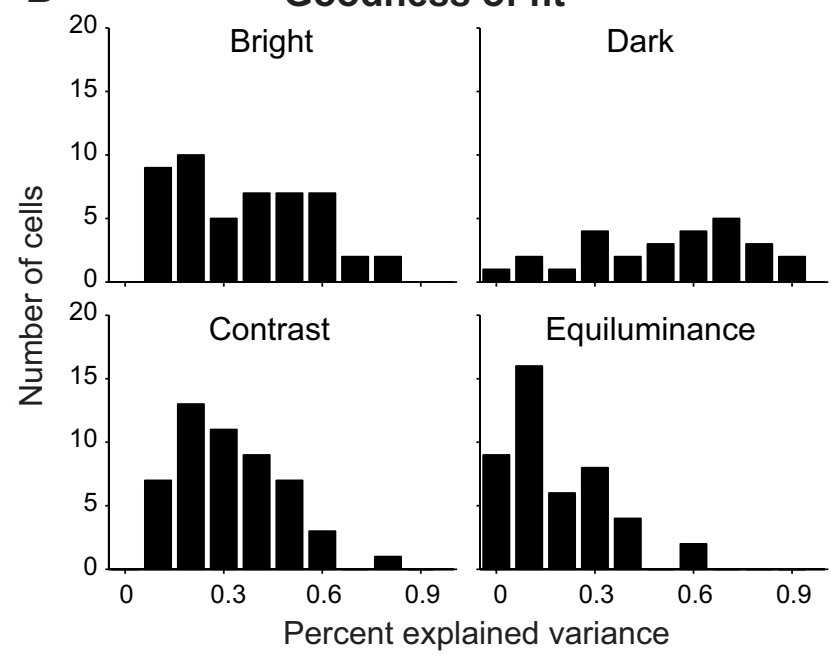

Figure 9. Color sensitivity indices of $\mathrm{V} 4$ neurons and the goodness of fit of linear cone excitation models with or without rectification. $\boldsymbol{A}$, Color sensitivity index, $\mathrm{Sl}$, is the ratio of the maximum response among equiluminant stimuli, Re, to the maximum response among achromatic stimuli, Ra. Each point shows $\mathrm{SI}$ for one cell. Color represents the classification from Figure 4. The dotted lines identify the range of sensitivity indices $(0.5<\mathrm{SI}<2.0)$ that were classified as color luminance cells by Johnson et al. (2001). Sensitivity indices of the vast majority of V4 neurons, except most equiluminance cells, fell in this range. Most equiluminance cells, however, had sensitivity indices $>2.0$. For 14 cells ( 3 bright, 1 dark, 9 equiluminance, and 1 flat cell), Ra was zero; these cells are shown at the right extreme (note broken $x$-axis). $\boldsymbol{B}$, Histogram of goodness of fit values for bright (top left), dark (top right), contrast (bottom left), and equiluminance (bottom right) cells. For each cell, we fit the neuronal responses with linear cone excitation models with or without rectification (see Materials and Methods). Goodness of fit ( $x$-axis) is given by the percentage of variance explained by the best fitting model. Some bright, dark, and contrast cells were well described by a rectified linear model, but for most equiluminance cells, linear cone excitation-based models provided a poor fit of the data (see Results). Mean goodness of fit for bright, dark, contrast, and equiluminance cells were 35.7, 50.1, 32.0, and 17.9 , respectively.

estimated the relative cone weights, $W_{\mathrm{L}}, W_{\mathrm{M}}$, and $W_{\mathrm{S}}$, that best predicted the observed responses. To allow for nonlinearities, we also considered models with half- and full-wave rectification (see Materials and Methods). For some neurons, such as the bright cell in Figure $2 \mathrm{~A}$, a half-wave rectified linear model provided an excellent fit to the observed responses, capturing $80 \%$ of the response variance. For this neuron, the $\mathrm{L}$ and $\mathrm{M}$ weights were nonopponent and the $S$ cone weights were small $\left(W_{\mathrm{L}}=0.37 ; W_{\mathrm{M}}=0.62 ; W_{\mathrm{S}}=\right.$ $-0.01)$. A half-wave rectified linear model also provided a good fit for the dark cell in Figure $2 B$ ( $88 \%$ explained variance). In this case, a large negative $\mathrm{M}$-cone weight $\left(W_{\mathrm{L}}=-0.02 ; W_{\mathrm{M}}=-0.9\right.$; $\left.W_{\mathrm{S}}=-0.08\right)$ successfully predicted strong responses to stimuli darker than the background and close to the red end of the color space. In contrast, the models considered here provided poor fits for the contrast and equiluminance cell responses in Figure 2, $C$ and $D$, capturing only 16 and $7 \%$ of the response variance, respectively. For the cell in Figure $2 D$, the best fitting model predicted a high baseline (7.4 spikes/s) and negative weights for all three cones $\left(W_{\mathrm{L}}=-0.08 ; W_{\mathrm{M}}=-0.85 ; W_{\mathrm{S}}=-0.07\right)$. Thus, predicted responses were highest for $C_{\text {lum }}=-50 \%$, intermediate for $C_{\text {lum }}=0$ and lowest for $C_{\text {lum }}=125 \%$. The linear cone weight model is particularly poor at explaining responses to equiluminance cells because, for these cells, the highest responses are at intermediate values for cone contrast. This produces a bellshaped relationship between the independent variables (cone contrasts) and the dependent variable (response) that cannot be successfully modeled by a linear weighting of cone contrasts. Results across the population are illustrated in Figure 9B. Linear cone excitation-based models, with or without rectification, provided a good fit for many bright and dark cells in our population, but fits were poor for a large fraction of the equiluminance cells. The mean percentage explained variance for the bright, dark, contrast, and equiluminance cells were 35.7, 50.1, 32.0, and 17.9, respectively. In terms of opponency, among the cells with significant linear fits (bright, 48 of 49; dark, 25 of 27; contrast, 51 of 51; equiluminance, 28 of 44 ), $60 \%$ of bright cells, $48 \%$ of dark, $31 \%$ of contrast, and $75 \%$ of equiluminance cells showed varying degrees of opponency in cone weights. Median cone weight ratio $\left(W_{\mathrm{L}} / W_{\mathrm{M}}\right)$ was -0.2 for bright and dark cells, 0.98 for contrast cells, and -0.7 for equiluminance cells that had significant linear fits. These results suggest that there was a trend toward weak opponency among bright and dark cells, no opponency among contrast cells, and balanced opponency among equiluminance cells, consistent with the observations based on the spread of SI indices above.

In summary, these comparisons suggest that a larger fraction of our V4 population displayed higher SI values than do V1 cells, largely as a result of the existence of equiluminance cells, and that while the responses of some $\mathrm{V} 4$ cells were well described by a linear cone model, many were not. The latter findings are consistent with reports from V1 and V2 that many color-selective neurons exhibit nonlinear combination of cone signals (Kiper et al., 1997; Hanazawa et al., 2000; Wachtler et al., 2003). Further studies are needed to develop more appropriate nonlinear models to describe and determine how V4 color responses arise, especially for equiluminance cells.

Coarse color and shape preferences of equiluminance cells To determine whether equiluminance cells differed from other V4 cells along stimulus dimensions other than luminance contrast, we compared cell categories in terms of coarse color (Figs. 10,11 ) and shape preferences (Fig. 12). More detailed characterization of the chromatic and shape preferences of V4 cells has been addressed in previous studies (Desimone and Schein, 1987; Schein and Desimone, 1990; Kobatake and Tanaka, 1994; Pasupathy and Connor, 2001; Kusunoki et al., 2006; Conway et al., 2007; Kotake et al., 2009); we simply ask whether the classes of cells defined here on the basis of luminance contrast response functions can be differentiated by their chromatic and shape preferences. For every cell, we characterized the numbers of colors that evoked responses greater than baseline $\left(N_{\text {col_sig }}\right)$ and greater than one-half of the maximum response $\left(N_{\text {col_hmax }}\right)$. 
A

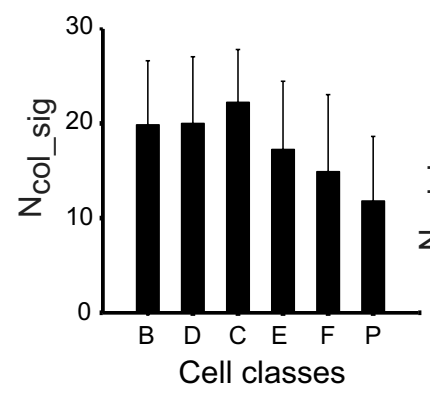

B

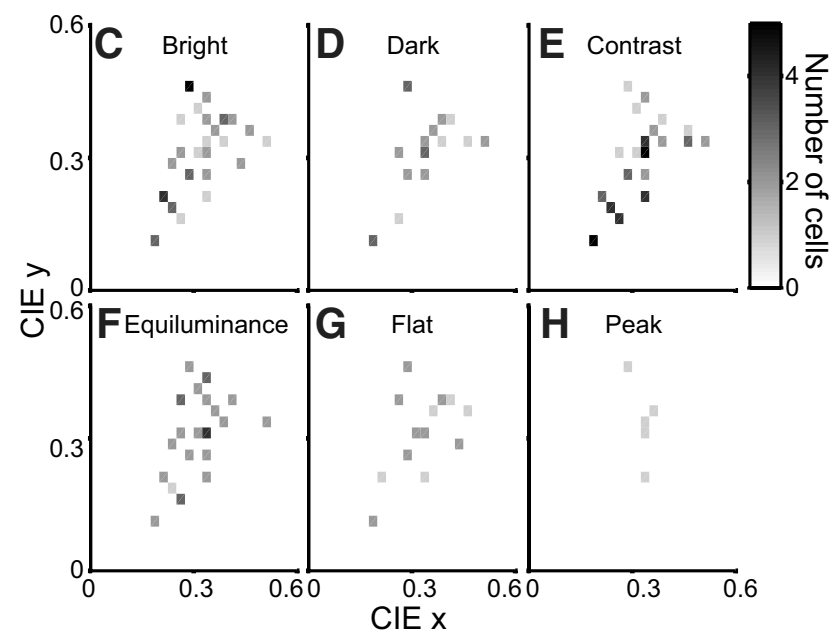

Figure 10. Color preferences across the V4 population. $A$, Mean and SD across cells for the number of colors (of 25) that elicited significant responses different from baseline for at least one of the four luminance contrasts tested; cells are grouped into categories (per Fig. 4) labeled here by the first letter of the category name: bright $(n=49)$, dark $(n=27)$, contrast $(n=51)$, equiluminance $(n=44)$, flat $(n=21)$, and peak cells $(n=5)$. $\boldsymbol{B}$, Number of colors that elicited more than half-maximum responses at the preferred contrast. $\mathbf{C}-\boldsymbol{H}$, Distribution of preferred colors that elicited the maximum response for neurons grouped by category. The $x$ - and $y$-axes correspond to CIExy coordinates. Grayscale indicates the number of cells that responded best to the corresponding color. In several cases, rectangles are white and not visible because no cell had a peak response at the corresponding color.

Across cells, $N_{\text {col_sig }}$ ranged between 2 and 25 (Fig. $10 \mathrm{~A}$ ), and the category averages ranged between 11 (for peak cells) and 22 (for contrast cells). For equiluminance cells, $N_{\text {col_sig }}$ was on average 17 colors (SD, 7.2), indicating that every equiluminance cell was driven by many colors and that the contrast function characterizations were not based on only a few colors. Average values for $N_{\text {col_hmax }}$ ranged between 6 and 14 (Fig. $10 \mathrm{~B}$ ), and for equiluminance cells was $8.8(\mathrm{SD}, 5)$. Thus, near equiluminance, roughly one-third of the colors evoked strong responses from equiluminance cells, suggesting that these cells were broadly color selective. Both measures, $N_{\text {col_sig }}$ and $N_{\text {col_hmax }}$, showed a significant dependence on the cell class (randomization one-way ANOVA, $p<0.01$ ), but the values observed for equiluminance cells were within the range of values observed across all classes of cells described here. To take into account the possibility that $N_{\text {col_hmax }}$ may be underestimated for equiluminance cells because low color contrast stimuli (at $0 \%$ luminance contrast) may be ineffective at driving these cells, we quantified $N_{\text {col_hmax_highcontrast }}$ based on only the 12 high color contrast stimuli (chromaticities 14-25; Fig. 1A) and our results were consistent with those reported above [i.e., $N_{\text {col_hmax_highcontrast }}$ showed significant dependence on cell class, but values exhibited by equiluminance cells $(4 \pm 2.5)$ were within the range across all cells (2-7)]. For all cell categories, preferred colors spanned the tested range (Fig. 10C$H$ ), consistent with previous results (Komatsu et al., 1992). Finally, we found no significant variation in peak firing rates, with or without baseline subtraction, across the cell classes (randomization ANOVA, $p<0.05$ ).

In terms of hue tuning, equiluminance cells can be broadly classified into three groups: (1) cells that show strong, unimodal tuning; (2) cells that show complex bimodal or multimodal tuning; and (3) cells that do not show statistically significant tuning. Figure 11 illustrates the hue response surfaces and tuning curves for six representative equiluminance cell examples. Hue response surfaces in the CIE color space (Fig. 11, images) were constructed by linear interpolation of normalized responses to stimuli at $0 \%$ luminance contrast. To assess the influence of color saturation on hue tuning, hue tuning curves were constructed by plotting response as a function of color direction relative to the achromatic point for each of the three bands of color contrasts tested (Fig. $1 A$ ) (see Materials and Methods). Figure $11 A-C$ shows examples that exhibited narrow, unimodal tuning in the CIE color space. The neuron in Figure $11 \mathrm{~A}$ responded preferentially to green colors at the highest color contrast (black line). Responses to mid and low color contrasts were weaker, but the peak position is similar. Responses at the highest color contrast showed statistically significant modulation as a function of color direction (Rayleigh test of circular uniformity, $p<0.05$, Bonferroni corrected). Strength of unimodal tuning was quantified as the resultant vector length (see Materials and Methods), which ranges from 0 (no unimodal tuning) to 1 (strong unimodal tuning). For the neuron in Figure $11 A$, the resultant vector length based on the responses to high color contrast stimuli was 0.72 . Figure $11, B$ and $C$, shows two other examples of equiluminance cells with strong unimodal tuning. Both neurons showed significant modulation of responses as a function of color direction at one or more color contrasts and the resultant vector length at the preferred color contrast was 0.5 and 0.74 , respectively.

Unlike the preceding examples, the hue tuning curves of examples D and E are more erratic and complex, but statistical tests indicate that these responses also showed significant modulation as a function of color direction at the mid (dark gray) and low (pale gray) color contrasts, respectively. The response surface of example D suggests that this neuron had two peaks in opposite color directions from the achromatic point, and this is reflected in the hue tuning curve for mid contrasts (dark gray); the resultant vector length was 0.2 consistent with the non-unimodal tuning curves. Example E, however, showed broad color tuning, responding to most equiluminant colors and the resultant vector length was again low (0.17). Finally, example F showed an annular response pattern in color space: responses were strong for all color directions with the exclusion of a small region centered on the achromatic point and the hue tuning in this case was not significantly different from uniform tuning.

Across our population, 18\% of equiluminance cells showed strong unimodal tuning like the examples A, B, and C (resultant vector length, $>0.4$ ) and $30 \%$ showed bimodal or multimodal tuning or broad and weaker unimodal tuning (as in examples D and E). The remaining 52\% of neurons (like example F) showed no significant response modulation as a function of hue. These proportions were similar to those observed for bright cells (16 and $27 \%$ for unimodal and multimodal, respectively), but the proportion of strongly unimodal cells was smaller among dark (8\%) and contrast cells $(2 \%)$.

Equiluminance cells are similar to the general V4 population in terms of the variety and complexity of their hue-tuning prop- 

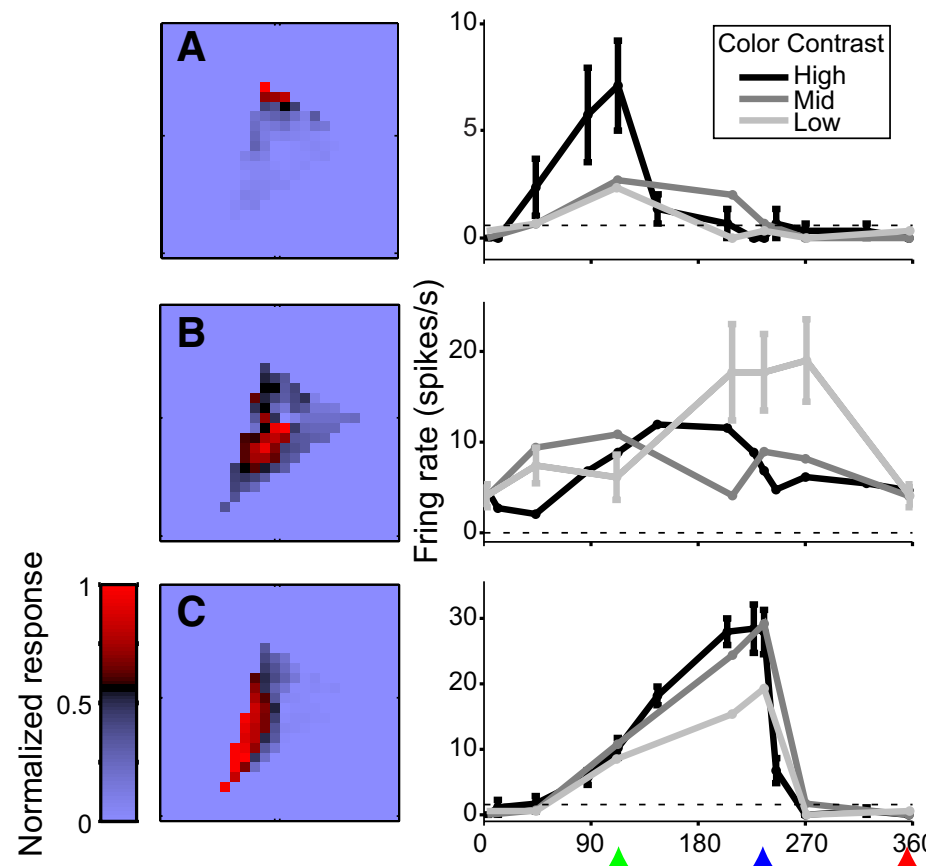
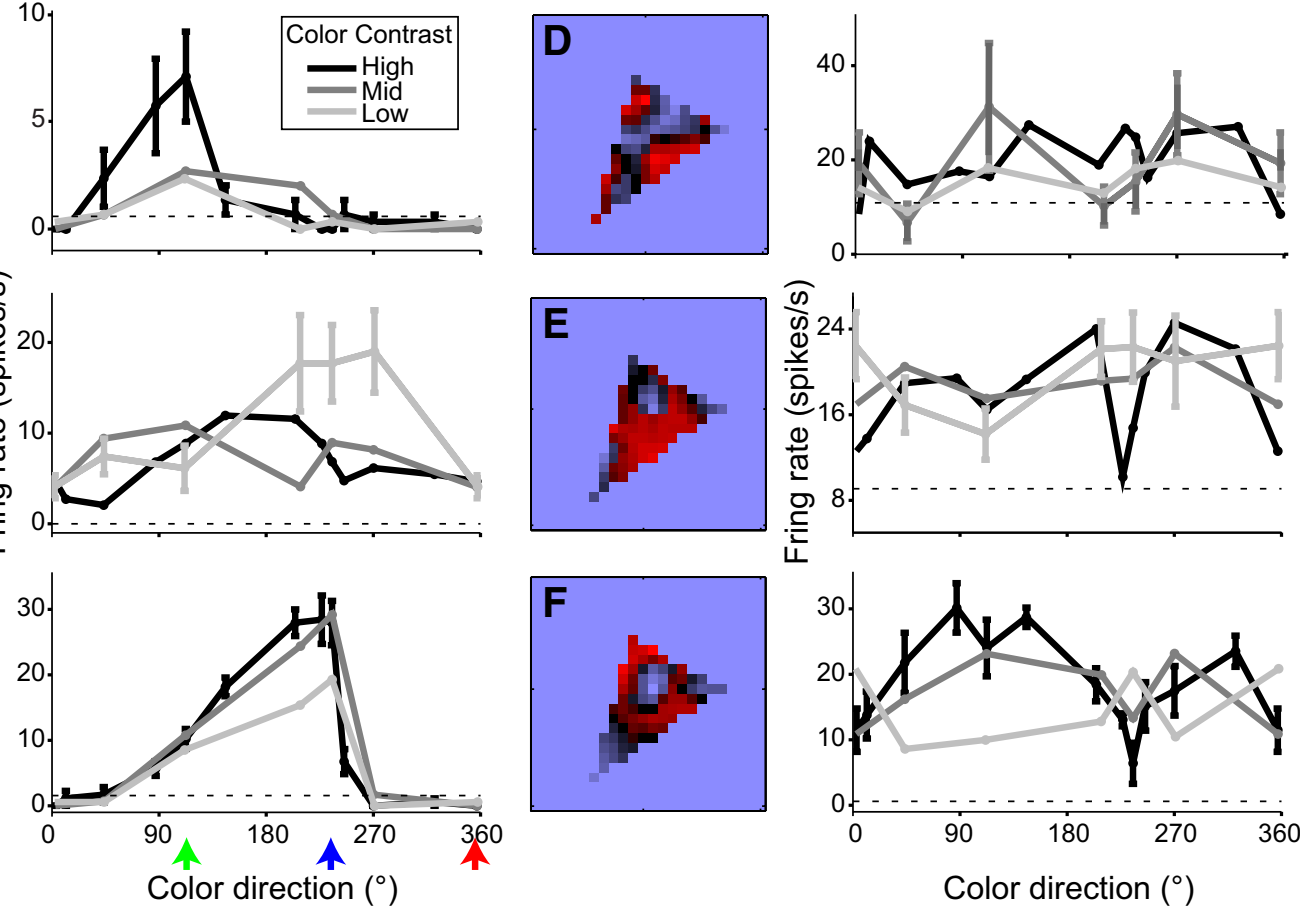

Figure 11. Hue tuning of six example equiluminance cells. $\boldsymbol{A}-\boldsymbol{F}$, Color images show the normalized, linearly interpolated response surface based on responses to stimuli at $0 \%$ luminance contrast. Color scale runs from blue to black to red representing low to high responses in the CIE space. Corresponding line plots show raw responses as a function of color direction in the CIE chromaticity diagram. Color direction ( $x$-axis) was measured relative to the achromatic point, $0^{\circ}$ is to the right, and angles increase counterclockwise. The red, green, and blue arrows mark the corresponding color directions. The pale gray, dark gray, and black lines represent responses to stimuli of low, mid, and high color contrasts, respectively. Error bars show SEM. The horizontal dashed line indicates the baseline response. Examples $\boldsymbol{A}-\boldsymbol{C}$ show unimodal tuning for hue at one or more saturations. Examples $\boldsymbol{D}$ and $\boldsymbol{E}$ also show statistically significant modulation of response as a function of color direction but lack unimodal hue tuning. Example $\boldsymbol{F}$ (same cell as in Fig. 3D) did not differ significantly from uniform tuning. See Results for details.

A
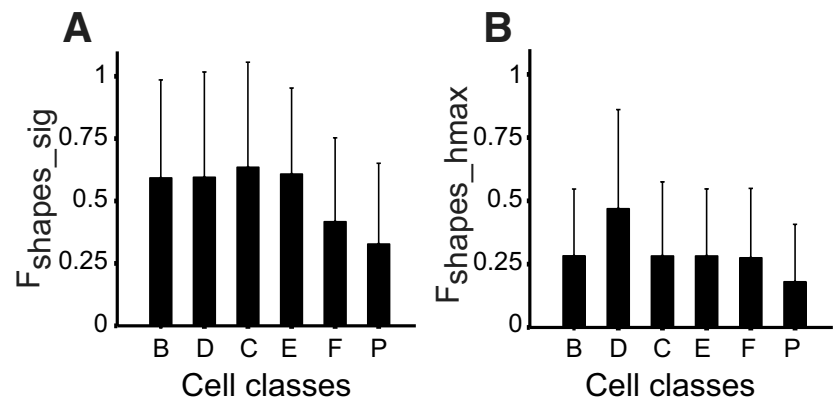

Figure 12. Shape preferences across the V4 population. $A$, Mean and SD across cells for the fraction of shapes that evoked responses significantly different from baseline. $\boldsymbol{B}$, Mean and SD for the fraction of shapes that evoked more than half-maximum responses. The $x$-axis shows first letter of cell class names, as in Figure $10 \mathrm{~A}$.

erties. Cells with narrow tuning for hue and saturation, like the cells in Figure $11 A-C$, have been previously reported in areas V1, V4, and IT cortex (Hanazawa et al., 2000; Kusunoki et al., 2006; Conway et al., 2007; Kotake et al., 2009). While equiluminance cells have not been previously reported, responses of an example cell from a glob region in the study by Conway et al. (2007), their Figure $6 A$, panel 2 , are consistent with those of a strongly huetuned equiluminance cell. Cells with tuning for multiple color directions or broad unimodal tuning have also been previously described in V4 (Kusunoki et al., 2006; Conway et al., 2007; Kotake et al., 2009). One previous V4 study investigated the consistency of color tuning across luminances by quantifying the correlation coefficient between responses to color stimuli at different luminances and found that $40 \%$ of interglob cells showed significant pairwise correlations for all comparisons (Conway et al., 2007). In our data set, only $10 \%$ of all cells showed significant pairwise correlations. This discrepancy might arise because we tested four luminances, and thus had six pairings, whereas they tested three luminances and had three pairings. Lack of significant correlation between responses at different luminances can result if (1) strong modulation by luminance contrast results in lack of responses at one or more of the tested luminances (for example, see Fig. 3D), or (2) chromatic tuning is dependent on luminance tuning such that the neuron prefers different chromaticities at different luminances. The SVD analysis (see Materials and Methods) directly tested which of these two reasons underlie the lack of correlation across some luminance pairings in our data. Because all except one of the neurons in our data set exhibited strong separability between the influence of chromaticity and luminance on neuronal responses, the lack of significant correlation across some luminance pairings is attributable to the former rather than the latter reason above. In summary, while colored stimuli are necessary to evoke strong responses from equiluminance cells, strong hue tuning is not a defining feature of these cells. Some equiluminance cells show strong hue tuning, but many cells show weak or no hue tuning consistent with the general V4 population.

To investigate whether stimulus shape modulates the responses of equiluminance cells and to assess differences in shape selectivity across cell classes, we examined the responses of a subset of 118 neurons that had been studied with a set of 10-29 shapes (Fig. $1 B$ ), each presented at multiple orientations. All classes of neurons showed similar levels of shape selectivity. On average, the fraction of stimuli that evoked significant responses $\left(F_{\text {shape_sig }}\right)$ ranged between 0.32 and 0.64 ; the fraction that evoked greater than half-maximum responses $\left(F_{\text {shape_hmax }}\right)$ ranged be- 
tween 0.18 and 0.47 . There were no systematic differences in $F_{\text {shape_sig }}$ and $F_{\text {shape_hmax }}$ across cell groups (randomization ANOVA). Among equiluminance cells, on average, $F_{\text {shape_sig }}$ was 0.61 and $F_{\text {shape_hmax }}$ was 0.29 . Thus, the different shapes used in this study modulated the responses of equiluminance cells and other V4 cells similarly. While this analysis does not characterize shape-tuning properties of these cells, it does suggest that the responses of equiluminance cells are modulated by, and could encode, information about visual shape. Again, maximum firing rate on the shape tuning test did not show a significant dependence on cell category.

\section{Discussion}

Equiluminance cells appear to represent a major physiological class of neuron in V4 that are similar to the general V4 population in terms of shape and color selectivity but quite unlike other established classes of cells anywhere in visual cortex in terms of their selectivity for luminance contrast. Specifically, equiluminance cells have strong responses near equiluminance and weaker responses at higher contrasts. Strong responses to equiluminant stimuli are not a surprising finding: many neurons in V1 and V2 respond strongly to equiluminant stimuli (Gouras and Kruger, 1979; Hubel and Livingstone, 1990; Lennie et al., 1990; Kiper et al., 1997). The novel and surprising finding here is that high luminance contrasts can suppress chromatic responses to levels substantially lower than those observed near equiluminance. To our knowledge, no previous study has characterized responses in visual cortex that monotonically decrease with increasing luminance contrast within the classical receptive field. Perhaps some equiluminance cells exist in V1 and V2; however, similar experiments, which modulated luminance contrast of color stimuli, have been conducted in V1 (Gouras and Kruger, 1979; Thorell et al., 1984; Hubel and Livingstone, 1990) and V2 (Kiper et al., 1997), and no such neurons have been described. Hubel and Livingstone (1990) have documented that a few neurons in layer $2 / 3$ of $\mathrm{V} 1$ show a shallow dip or no dip at all at equiluminance, analogous to flat cells described here. Apart from that, in analogous studies, V1 and V2 responses increased with the incremental addition of luminance to chromatic stimuli (Gouras and Kruger, 1979; Thorell et al., 1984; Kiper et al., 1997). While it is possible that V4 inherits equiluminance selectivity from other areas, given the absence of such cells in previous detailed studies of color selectivity in V1 and V2, it seems more likely that the response properties of V4 equiluminance cells emerge de novo in V4.

It has long been hypothesized that double-opponent cells, which show chromatic and spatial opponency, play an important role in encoding simultaneous color contrast and chromatically defined form (Daw, 1967; Livingstone and Hubel, 1984; Michael, 1985; Shapley and Hawken, 2002; Conway, 2009). The precise definition and RF structure of double opponent cells has been widely debated: while one view is that they compose $\sim 5-10 \%$ of V1 and have coarse receptive field structure (Conway, 2001; Conway and Livingstone, 2006), others argue that they compose $\sim 30 \%$ of V1 (color luminance cells; Johnson et al., 2001) and that they are well equipped for form vision (Shapley and Hawken, 2002). Regardless of how their RFs arise and whether they fit the precise definition of double opponency, these color luminance cells respond well to achromatic luminance and equiluminant stimuli (Lennie et al., 1990; Johnson et al., 2001; Wachtler et al., 2003). They are tuned to stimulus orientation and high spatial frequencies (Johnson et al., 2001) making them well suited to play a role in encoding object boundaries defined by chromatic contrast, luminance contrast, or both (Shapley and Hawken, 2002). The bright, dark, contrast, flat, and peak cells in our population have response properties similar to color luminance cells in V1-these cells show selective responses to complex shapes and respond equally well to equiluminant and achromatic stimuli. Thus, the response properties of these neurons could be derived from color luminance cells in V1.

A small fraction, $\sim 10 \%$, of $\mathrm{V} 1$ neurons respond stronger to equiluminant stimuli than to luminance stimuli (Lennie et al., 1990; Johnson et al., 2001). But because these color-sensitive neurons typically have low-pass spatial frequency tuning curves and respond well to extended chromatic surfaces, the properties of V4 equiluminance cells are unlikely to be derived solely from these V1 responses. Responses of equiluminance cells to their preferred colors weaken when those chromaticities are presented at higher luminance contrasts. Therefore, linear models based on cone excitation or contrast fail to explain their responses (see Results). Response properties of equiluminance cells could arise by combining excitatory chromatic inputs with inhibitory inputs that elevate with increasing contrast. For example, equiluminance cells could receive an especially strong inhibition akin to contrast normalization. To test for this, we superimposed high-contrast achromatic gratings of varying spatial frequencies on preferred color equiluminant stimuli and found that this did not reproduce the suppression we observed by increasing the luminance contrast of the shape (data not shown). This suggests that patternspecific suppressive mechanisms are involved in the construction of equiluminance cells, perhaps requiring coextensive luminance and color gradients. It is implausible that our results are due to luminance artifacts generated by chromatic aberration, because equiluminance cells show unimodal response curves and do not respond to high luminance contrasts. In other words, being off by a modest amount from ideal equiluminance for each cell would not change the overall interpretation of our data. Past studies have demonstrated that V4 color responses can be influenced by task requirements and top-down signals including attention (Motter, 1994; Ogawa and Komatsu, 2004; Bichot et al., 2005). Such influences cannot explain the different categories of neurons reported here because these animals were not trained on any task other than passive fixation and the proportions of different categories of neurons were similar for the two animals.

Several psychophysical studies have investigated the extent of segregation and overlap in the processing of chromatic and luminance stimuli. Evidence from experiments using achromatic luminance and equiluminant color stimuli (but not their combination) support the existence of a color-based shape analysis system that is independent of luminance information. Because simultaneous and opposite spatial frequency shifts (Favreau and Cavanagh, 1981) and tilt aftereffects (Flanagan et al., 1990) were obtained in adaptation experiments with equiluminant-chromatic and achromatic gratings, it has been argued that a channel exists that codes spatial frequency and orientation solely based on chromatic information. Results from adaptation experiments measuring detection thresholds (Bradley et al., 1988; Murasugi and Cavanagh, 1988) are consistent with this hypothesis, and results from visual search experiments (Cavanagh et al., 1990) indicate that orientation and size are basic coding dimensions for equiluminous colors. These results point to the existence of a chrominance channel that analyzes shape information solely based on chromatic signals (Cavanagh, 1991). More extensive investigation along multiple directions in the chrominance by luminance space supports the existence of multiple color mechanisms, not just a second chrominance-based 
mechanism (Gegenfurtner and Kiper, 1992; Hansen and Gegenfurtner, 2006). Results from these studies, which test contrast detection and image segmentation in the presence of noise, reveal that behavioral performance is influenced by noise over a narrow range centered on the stimulus direction in the chrominance $X$ luminance space. This supports the hypothesis of multiple color mechanisms with peaks at different chrominance $\times$ luminance combinations; the narrow tuning widths suggest interaction between linear mechanisms.

Equiluminance cells in V4 have properties that correspond well with the requirements of the shape-encoding chrominance channel proposed above: (1) they are the first set of visual neurons that have been shown to be selective for low luminance contrasts, where shape information is largely based on color contrast; (2) they are shape selective; and (3) they form a sizable subpopulation of V4. The narrow tuning of equiluminance cells along the luminance contrast dimension (Fig. 8) is qualitatively consistent with the narrow tuning width observed in the psychophysical experiments described above, thus lending physiological support for the proposal of multiple color mechanisms. Because equiluminant boundaries are more common than previously thought and they provide a statistically independent source of shape information in natural images (Hansen and Gegenfurtner, 2009), form information carried by equiluminance cells may play an important role in shape perception.

In conclusion, our results suggest that there is a particular class of cells in V4 that is specialized to represent and process near-equiluminant visual scenes, in which the information is largely based on chromatic contrast. These equiluminance cells are well suited to play a role in perception near equiluminance and could be crucial for widely cited color tasks of foraging for ripe fruit among green foliage and defeating luminance-matched camouflage. Our findings suggest that the representation of scenes based on chromatic signals may be a specialization in V4 and supports the hypothesis that form at equiluminance and form at higher contrasts are processed in separate channels $(\mathrm{Ca}-$ vanagh, 1991; Gegenfurtner and Kiper, 1992; Hansen and Gegenfurtner, 2006). Finally, these results motivate a host of questions, including how this selectivity for equiluminance is generated, and how the signals studied here are used within and beyond V4, which are likely to inspire further experiments and fuel heated debates that have long marked the study of color vision (De Valois, 1960).

\section{References}

Albrecht DG (1995) Visual cortex neurons in monkey and cat: effect of contrast on the spatial and temporal phase transfer functions. Vis Neurosci 12:1191-1210.

Bichot NP, Rossi AF, Desimone R (2005) Parallel and serial neural mechanisms for visual search in macaque area V4. Science 308:529-534.

Bradley A, Switkes E, De Valois K (1988) Orientation and spatial frequency selectivity of adaptation to color and luminance gratings. Vision Res 28:841-856.

Bushnell BN, Harding PJ, Kosai Y, Pasupathy A (2011) Partial occlusion modulates contour-based shape encoding in primate area V4. J Neurosci 31:4012-4024.

Carandini M, Heeger DJ, Movshon JA (1997) Linearity and normalization in simple cells of the macaque primary visual cortex. J Neurosci 17:8621-8644.

Cavanagh P (1991) Vision at equiluminance. In: Vision and visual dysfunction: limits of vision (Kulikowski JJ, Murray IJ, Walsh V, eds), pp 234250. Boca-Raton, FL: CRC.

Cavanagh P, Arguin M, Treisman A (1990) Effect of surface medium on visual search for orientation and size features. J Exp Psychol Hum Percept Perform 16:479-491.
Chaparro A, Stromeyer CF 3rd, Huang EP, Kronauer RE, Eskew RT Jr (1993) Colour is what the eye sees best. Nature 361:348-350.

Cole GR, Hine T (1992) Computation of cone contrasts for color vision. Behav Res Methods Instrum Comput 24:22-27.

Conway BR (2001) Spatial structure of cone inputs to color cells in alert macaque primary visual cortex (V-1). J Neurosci 21:2768-2783.

Conway BR (2009) Color vision, cones, and color-coding in the cortex. Neuroscientist 15:274-290.

Conway BR, Livingstone MS (2006) Spatial and temporal properties of cone signals in alert macaque primary visual cortex. J Neurosci 26:1082610846.

Conway BR, Moeller S, Tsao DY (2007) Specialized color modules in macaque extrastriate cortex. Neuron 56:560-573.

Daw NW (1967) Goldfish retina: organization for simultaneous color contrast. Science 158:942-944.

Depireux DA, Simon JZ, Klein DJ, Shamma SA (2001) Spectro-temporal response field characterization with dynamic ripples in ferret primary auditory cortex. J Neurophysiol 85:1220-1234.

Desimone R, Schein SJ (1987) Visual properties of neurons in area V4 of the macaque: sensitivity to stimulus form. J Neurophysiol 57:835-868.

De Valois RL (1960) Color vision mechanisms in the monkey. J Gen Physiol 43 [Suppl]:115-128.

Favreau OE, Cavanagh P (1981) Color and luminance: independent frequency shifts. Science 212:831-832.

Flanagan P, Cavanagh P, Favreau OE (1990) Independent orientationselective mechanisms for the cardinal directions of colour space. Vision Res 30:769-778.

Gawne TJ, Kjaer TW, Richmond BJ (1996) Latency: another potential code for feature binding in striate cortex. J Neurophysiol 76:1356-1360.

Gegenfurtner KR, Kiper DC (1992) Contrast detection in luminance and chromatic noise. J Opt Soc Am A 9:1880-1888.

Gegenfurtner KR, Kiper DC, Levitt JB (1997) Functional properties of neurons in macaque area V3. J Neurophysiol 77:1906-1923.

Gouras P, Krüger J (1979) Responses of cells in foveal visual cortex of the monkey to pure color contrast. J Neurophysiol 42:850-860.

Hanazawa A, Komatsu H, Murakami I (2000) Neural selectivity for hue and saturation of colour in the primary visual cortex of the monkey. Eur J Neurosci 12:1753-1763.

Hansen T, Gegenfurtner KR (2006) Higher level chromatic mechanisms for image segmentation. J Vis 6:239-259.

Hansen T, Gegenfurtner KR (2007) Higher order color mechanisms for image segmentation. In: Brain, vision and artificial intelligence LNCS (Mele F, Ramella G, Santillo S, Ventriglia F, eds), pp 72-83. Heidelberg: Springer.

Hansen T, Gegenfurtner KR (2009) Independence of color and luminance edges in natural scenes. Vis Neurosci 26:35-49.

Hubel DH, Livingstone MS (1990) Color and contrast sensitivity in the lateral geniculate body and primary visual cortex of the macaque monkey. J Neurosci 10:2223-2237.

Johnson EN, Hawken MJ, Shapley R (2001) The spatial transformation of color in the primary visual cortex of the macaque monkey. Nat Neurosci 4:409-416.

Kiper DC, Fenstemaker SB, Gegenfurtner KR (1997) Chromatic properties of neurons in macaque area V2. Vis Neurosci 14:1061-1072.

Kobatake E, Tanaka K (1994) Neuronal selectivities to complex object features in the ventral visual pathway of the macaque cerebral cortex. J Neurophysiol 71:856-867.

Komatsu H, Ideura Y, Kaji S, Yamane S (1992) Color selectivity of neurons in the inferior temporal cortex of the awake macaque monkey. J Neurosci 12:408-424.

Kotake Y, Morimoto H, Okazaki Y, Fujita I, Tamura H (2009) Organization of color-selective neurons in macaque visual area V4. J Neurophysiol 102:15-27.

Kusunoki M, Moutoussis K, Zeki S (2006) Effect of background colors on the tuning of color-selective cells in monkey area V4. J Neurophysiol 95:3047-3059.

Lee J, Williford T, Maunsell JH (2007) Spatial attention and the latency of neuronal responses in macaque area V4. J Neurosci 27:9632-9637.

Lennie P, Krauskopf J, Sclar G (1990) Chromatic mechanisms in striate cortex of macaque. J Neurosci 10:649-669.

Livingstone MS, Hubel DH (1984) Anatomy and physiology of a color system in the primate visual cortex. J Neurosci 4:309-356. 
Maunsell JH, Ghose GM, Assad JA, McAdams CJ, Boudreau CE, Noerager BD (1999) Visual response latencies of magnocellular and parvocellular LGN neurons in macaque monkeys. Vis Neurosci 16:1-14.

Mazer JA, Vinje WE, McDermott J, Schiller PH, Gallant JL (2002) Spatial frequency and orientation tuning dynamics in area V1. Proc Natl Acad Sci U S A 99:1645-1650.

Michael CR (1985) Laminar segregation of color cells in the monkey's striate cortex. Vision Res 25:415-423.

Motter BC (1994) Neural correlates of attentive selection for color or luminance in extrastriate area V4. J Neurosci 14:2178-2189.

Murasugi CM, Cavanagh P (1988) Anisotropy in the chromatic channel: a horizontal-vertical effect. Spat Vis 3:281-291.

Ogawa T, Komatsu H (2004) Target selection in area V4 during multidimensional visual search task. J Neurosci 24:6371-6382.

Pasupathy A, Connor CE (2001) Shape representation in area V4: positionspecific tuning for boundary conformation. J Neurophysiol 86:25052519.

Peña JL, Konishi M (2001) Auditory spatial receptive fields created by multiplication. Science 292:249-252.
Peng X, Van Essen DC (2005) Peaked encoding of relative luminance in macaque areas V1 and V2. J Neurophysiol 93:1620-1632.

Reynolds JH, Pasternak T, Desimone R (2000) Attention increases sensitivity of V4 neurons. Neuron 26:703-714.

Schein SJ, Desimone R (1990) Spectral properties of V4 neurons in the macaque. J Neurosci 10:3369-3389.

Sclar G, Maunsell JH, Lennie P (1990) Coding of image contrast in central visual pathways of the macaque monkey. Vision Res 30:1-10.

Shapley R, Hawken M (2002) Neural mechanisms for color perception in the primary visual cortex. Curr Opin Neurobiol 12:426-432.

Smith VC, Pokorny J (1975) Spectral sensitivity of the foveal cone pigments between 400 and $500 \mathrm{~nm}$. Vision Res 15:161-171.

Thorell LG, De Valois RL, Albrecht DG (1984) Spatial mapping of monkey V1 cells with pure color and luminance stimuli. Vision Res 24:751-769.

Wachtler T, Sejnowski TJ, Albright TD (2003) Representation of color stimuli in awake macaque primary visual cortex. Neuron 37:681-691.

Zeki SM (1973) Colour coding in rhesus monkey prestriate cortex. Brain Res 53:422-427. 